ANL- 6373

Physics

(TID-4500, 16th Ed.)

AEC Research and

Development Report

ARGONNE NATIONAL LABORATORY

9700 South Cass Avenue

Argonne, Illinois

\title{
THEORETICAL REACTION CROSS SECTIONS \\ FOR ALPHA PARTICLES WITH AN \\ OPTICAL MODEL
}

by

J.R. Huizenga* and G. J.Igo**

\footnotetext{
*Chemistry Division

** Lawrence Radiation Laboratory, Berkeley, California
}

May 1961

Operated by The Univèrsity of Chicago under

Contract W-31-109-eng-38 


\section{DISCLAIMER}

This report was prepared as an account of work sponsored by an agency of the United States Government. Neither the United States Government nor any agency Thereof, nor any of their employees, makes any warranty, express or implied, or assumes any legal liability or responsibility for the accuracy, completeness, or usefulness of any information, apparatus, product, or process disclosed, or represents that its use would not infringe privately owned rights. Reference herein to any specific commercial product, process, or service by trade name, trademark, manufacturer, or otherwise does not necessarily constitute or imply its endorsement, recommendation, or favoring by the United States Government or any agency thereof. The views and opinions of authors expressed herein do not necessarily state or reflect those of the United States Government or any agency thereof. 


\section{DISCLAIMER}

Portions of this document may be illegible in electronic image products. Images are produced from the best available original document. 
TABLE OF CONTENTS

Page

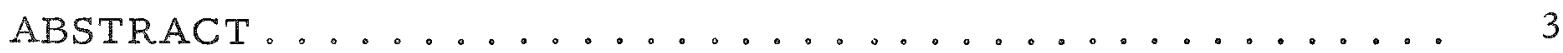

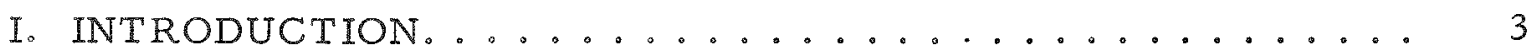

II. RESULTS AND DISCUSSION ................... 4

A. Simplified Calculation of Reaction Cross Sections with a Parabolic Approximation of Optical Model Real Potential..

B. Comparison of Reaction Cross Sections from Various

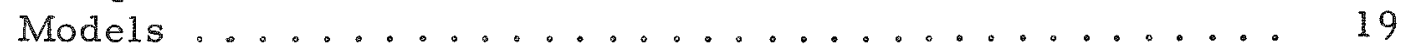

C. Optical-model Cross-section Dependence on Nuclear

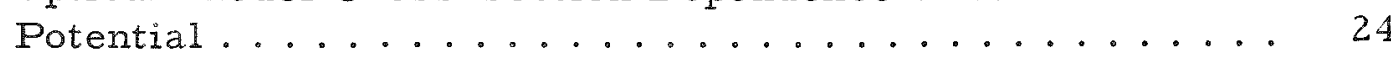

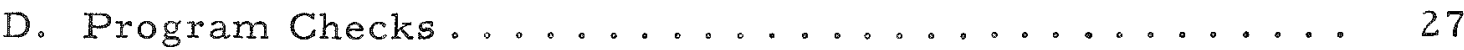

III. ACKNOWLEDGMENTS .................. 27

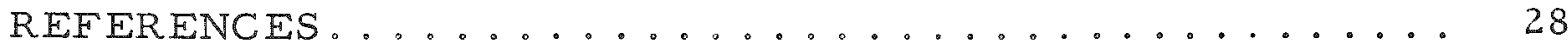




\title{
THEORETICAL REACTION CROSS SECTIONS \\ FOR ALPHA PARTICLES WITH AN \\ OPTICAL MODEL
}

by

J.R. Huizenga and G.J.Igo

\begin{abstract}
The transmission coefficients $T_{p}$ and total reaction cross sections $\sigma_{\mathrm{R}}$ for alpha particles in the energy range $0-46 \mathrm{Mev}$ interacting with twenty target nuclei with atomic numbers ranging from 10 to 92 arecalculated with anoptical model program in which a previously determined complex nuclear potential is utilized. The dependence of the $T_{l}$ ralues, and hence of $\sigma_{R}$, on the Woods-Saxon parameters is investigated as a function of projectile energy. The optical model reaction cross sections are compared with those derived from (1) a square-well potential and (2) a model which approximates the real optical model potential barriex by a parabola and makes use of the Hill-Wheeler penetration formula for a parabolic potential.
\end{abstract}

\section{INTRODUCTION}

Shapiro(1) and Blatt and Weisskopf $(2)$ have calculated total reaction cross sections for alpha particles with a square-well potential. The total reaction cross section is given by

$$
\sigma_{R}=\pi \lambda^{2} \sum_{h=0}^{\infty}(2 \ell+1) \mathrm{T}_{b}(\epsilon) \quad
$$

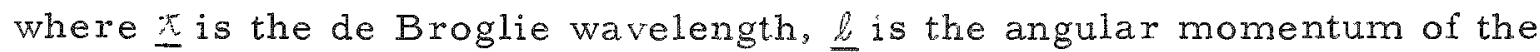
incident particle in units of $\bar{h}$, and $T(\bar{\epsilon})$ is the transmission coefficient of the incident particle of energy $\subseteq$. The transmission coefficients (actually $4 / T_{\ell}$ are tabulated) for alpha particles derived from the square-well potential are available(3) for target nuclei with $Z<40$. Igo(4) has calculated total reaction cross sections with a complex nuclear potential for six target nuclei with alpha-particle projectiles of a few energies. Transmission coefficients calculated with the complex nuclear potential are not available. 
Since alpha-particle transmission coefficients for high-Z targets are not available, we have calculated this quantity for a large variety of targets at a number of alpha-particle energies with a complex nuclear potential. For the light-Z $\underline{Z}$ targets, the transmission coefficients derived with the complex nuclear potential of the optical model are compared with those previously calculated with the square-well potential.(3)

\section{RESULTS AND DISCUSSION}

The optical-model potential employed in these calculations is written as

$$
V_{h}(x)=V_{c}+\frac{\ell(y+1) h^{2}}{2 M_{1} x^{2}}+\frac{V}{1+\exp \left(\frac{r-x_{0}}{d}\right)}+\frac{i W}{1+\exp \left(\frac{r-x_{0}}{d}\right)}
$$

where $V_{c}$ is the Coulomb potential, $M_{\alpha}$ the reduced mass of the alpha particle, and the third and fourth terms (in units of $\mathrm{Mev}$ ) are the real and imaginary parts of the alpha-nucleus potential, respectively, exclusive of the Coulomb and centrifugal potentials. The Coulomb potential of the HillFord (5) charge distribution was employed in Eq. (2). For alpha particles this is given by

$$
V_{c}=\frac{2 Z e^{2}}{r_{c}}\left[\frac{1}{n^{2}}+\frac{1}{2}-\frac{x^{2}}{6}+\frac{e^{-n}}{n^{2}}\left(\frac{1-e^{n x}}{n x}+\frac{e^{n x}}{2}\right)\right] /\left(\frac{1}{3}+\frac{2}{n^{2}}+\frac{e^{-n}}{n^{3}}\right)(\text { for } x<1)
$$

and

$$
V_{c}=\frac{2 Z e^{2}}{r_{c}}\left\{\frac{1}{x}-\left[\frac{\left(\frac{1}{x}+\frac{n}{2}\right) e^{n-n x}}{e^{-n}+2 n+\frac{1}{3} n^{3}}\right]\right\}(\text { for } x>1)
$$

where $\underline{x}=r / r_{C}$ and where $\underline{n}$ is 10 for heavy elements and is proportional to $\mathrm{A}^{1 / 3}$. The quantity ${ }^{r_{C}}$ is the distance out to the half-value point of the charge distribution. The value $1.17 \mathrm{~A}^{1 / 3} \times 10^{-13} \mathrm{~cm}$ for $\mathrm{r}_{\mathrm{C}}$ was chosen larger than the value obtained from the electron-scattering experiments $(6)$ to take into account roughly the effect of the finite size of the alphaparticle charge distribution.

The parameters $V, W, r_{0}$, and $\underline{d}$ in the Woods-Saxon potential, (7)

$$
(V+i W) /\left[1+\exp \left(\frac{r-r_{0}}{d}\right)\right] \text {, }
$$


were chosen to reproduce the nuclear potential given by $(4)$

$$
-1100 \exp \left[-\left(\frac{r-1.17 A^{1 / 3}}{0.574}\right)\right]-45.7 i \exp \left[-\left(\frac{x-1.40 A^{1 / 3}}{0.578}\right)\right]
$$

for values of $\underline{r}$ where the real part of the nuclear potential is greater than $-10 \mathrm{Mev}$. The Woods-Saxon parameters for the nuclides which we investigated are given in Table $I$. The depth of the potential for small values of $\underline{r}$ is also fixed when the parameters of Table I are used, although it has been shown that alpha-particle scattering is not sensitive to the potential depth.

The total reaction cross sections $\sigma_{R}$ and the transmission coef ficients $T_{\ell}$ calculated with the parameters of $T$ able $I$ are listed in Tables II and III, respectively. It should be emphasized that the alphaparticle energies are given in the laboratory system.

Table I

WOODS-SAXON POTENTIAL PARAMETERS"

\begin{tabular}{|c|c|c|c|c|c|}
\hline Nuclide & W(Mev) & $n$ & Nuclide & W(Mev) & $n$ \\
\hline $10 \mathrm{Ne}^{20}$ & -5.30 & 4.42 & $37 \mathrm{Rb}^{85}$ & -13.30 & 7.16 \\
\hline $19 \mathrm{~K}^{41}$ & -8.70 & 5.60 & ${ }_{41} \mathrm{Nb}^{93}$ & -13.74 & 7.37 \\
\hline $22^{\mathrm{Ti}^{48}}$ & -9.51 & 5.91 & ${ }_{45} \mathrm{Rh}^{103}$ & -14.30 & 7.62 \\
\hline${ }_{23} v^{51}$ & -10.00 & 6.04 & $505 n^{119}$ & -16.20 & 8.00 \\
\hline${ }_{24} \mathrm{Cr}^{52}$ & -9.90 & 6.07 & $60^{\mathrm{Nd}^{144}}$ & -18.23 & 8.53 \\
\hline${ }_{25} \mathrm{Mn}^{53}$ & -9.98 & 6.11 & $70^{Y b^{173}}$ & -20.79 & 9.07 \\
\hline${ }_{2} 5^{M n^{55}}$ & -10.25 & 6.19 & $78^{19 t^{195}}$ & -22.10 & 9.44 \\
\hline $26 \mathrm{Fe}^{56}$ & -10.26 & 6.23 & $82 \mathrm{~Pb}^{206}$ & -23.00 & 9.61 \\
\hline${ }_{27} \mathrm{Co}^{55}$ & -10.17 & 6.19 & $90^{T h}{ }^{232}$ & -26.11 & 10.00 \\
\hline $32^{\mathrm{Ge}^{72}}$ & -11.85 & 6.77 & ${ }_{92} U^{235}$ & -27.00 & 10.04 \\
\hline
\end{tabular}

"In all cases,

$V($ Mev $)=-50$

$r_{0}($ fermi $)=1.17 \mathrm{~A}^{1 / 3}+1.77$

$r_{C}(f e r m i)=1.17 \mathrm{~A}^{1 / 3}$

and

$d($ fermi $)=0.576$ 
Table II

THEORETICAL REACTION CROSS SECTIONS $(\sigma R$ ) MLLIBARNS

\begin{tabular}{|c|c|c|c|c|c|c|c|c|c|c|c|}
\hline $\begin{array}{l}\text { Elab } \\
\text { (Mev) }\end{array}$ & $10^{\mathrm{N} \mathrm{N}^{20}}$ & $19^{41}$ & $22^{\pi^{48}}$ & $23^{51}$ & $24 \mathrm{Cr}^{52}$ & ${ }_{25} \mathrm{Mn}^{53}$ & $25^{\mathrm{Mn}} \mathrm{n}^{55}$ & $26 \mathrm{Fe}^{56}$ & ${ }_{27} \mathrm{C}^{55}$ & $326 \mathrm{e}^{72}$ & $37^{R b^{85}}$ \\
\hline $\begin{array}{l}2 \\
3\end{array}$ & $\begin{array}{l}0.36 \\
23.1\end{array}$ & & & & & & & & & & \\
\hline $\begin{array}{l}4 \\
6\end{array}$ & $\begin{array}{l}177 \\
608\end{array}$ & $\begin{array}{l}0.44 \\
70\end{array}$ & $\begin{array}{l}0.035 \\
16.9\end{array}$ & $\begin{array}{l}0.016 \\
10.5\end{array}$ & $\begin{array}{l}0.0059 \\
5.49\end{array}$ & $\begin{array}{l}0.0022 \\
2.85\end{array}$ & $\begin{array}{l}0.0026 \\
3.28\end{array}$ & $\begin{array}{l}0.0010 \\
1.68\end{array}$ & $\begin{array}{l}0.0003 \\
0.741\end{array}$ & 0.053 & 0.0027 \\
\hline $\begin{array}{l}7 \\
8\end{array}$ & $\begin{array}{l}745 \\
862\end{array}$ & 422 & $\begin{array}{l}91.5 \\
250\end{array}$ & 205 & $\begin{array}{l}40.5 \\
150\end{array}$ & $\begin{array}{l}24.2 \\
105\end{array}$ & 116 & $\begin{array}{l}16.0 \\
79.0\end{array}$ & $\begin{array}{l}8.05 \\
46.5\end{array}$ & 8.17 & 0.91 \\
\hline 19 & 1017 & 744 & $\begin{array}{l}435 \\
603\end{array}$ & 562 & $\begin{array}{l}320 \\
496\end{array}$ & $\begin{array}{l}257 \\
431\end{array}$ & 452 & $\begin{array}{l}216 \\
388\end{array}$ & $\begin{array}{l}151 \\
307\end{array}$ & 140 & 35.0 \\
\hline $\begin{array}{l}11 \\
12\end{array}$ & & 961 & $\begin{array}{l}745 \\
862\end{array}$ & 834 & $\begin{array}{l}650 \\
780\end{array}$ & $\begin{array}{l}589 \\
725\end{array}$ & 749 & $\begin{array}{l}551 \\
693\end{array}$ & $\begin{array}{l}469 \\
614\end{array}$ & 454 & 249 \\
\hline 13 & & & 959 & & 888 & 839 & & 813 & 739 & & \\
\hline 14 & 1128 & 1104 & 1040 & 1024 & 980 & 935 & 960 & 914 & 844 & 736 & 555 \\
\hline $\begin{array}{l}16 \\
18\end{array}$ & 1175 & 1280 & & $\begin{array}{l}1159 \\
1259\end{array}$ & & & $\begin{array}{l}1112 \\
1224\end{array}$ & & & 1108 & $\begin{array}{l}809 \\
1005\end{array}$ \\
\hline $\begin{array}{l}20 \\
22\end{array}$ & 1170 & 1376 & & $\begin{array}{l}1334 \\
1393\end{array}$ & & & $\begin{array}{l}1310 \\
1377\end{array}$ & & & 1329 & 1278 \\
\hline $\begin{array}{l}24 \\
26\end{array}$ & 1200 & 1429 & & $\begin{array}{l}1438 \\
1473\end{array}$ & & & 1459 & & & 1471 & 1455 \\
\hline $\begin{array}{l}28 \\
30\end{array}$ & 1186 & 1461 & & $\begin{array}{l}1501 \\
1524\end{array}$ & & & 1529 & & & 1566 & 1561 \\
\hline $\begin{array}{l}34 \\
38\end{array}$ & 1140 & $\begin{array}{l}1482 \\
1495\end{array}$ & & $\begin{array}{l}1559 \\
1581\end{array}$ & & & $\begin{array}{l}1570 \\
1599\end{array}$ & & & $\begin{array}{l}1633 \\
1681\end{array}$ & $\begin{array}{l}1663 \\
1727\end{array}$ \\
\hline $\begin{array}{l}42 \\
46\end{array}$ & 1081 & $\begin{array}{l}1495 \\
1493\end{array}$ & & $\begin{array}{l}1595 \\
1603\end{array}$ & & & $\begin{array}{l}1618 \\
1628\end{array}$ & & & $\begin{array}{l}1716 \\
1742\end{array}$ & $\begin{array}{l}1774 \\
1811\end{array}$ \\
\hline
\end{tabular}

\begin{tabular}{|c|c|c|c|c|c|c|c|c|c|}
\hline $\begin{array}{l}E_{\text {Lab }} \\
\text { (Mev) }\end{array}$ & $41^{\mathrm{N} 0^{93}}$ & ${ }_{45} \mathrm{Rh}^{103}$ & ${ }_{50} \mathrm{Sn}^{119}$ & $60 \mathrm{Nd}^{144}$ & ${ }_{70} \mathrm{Y}_{6}{ }^{173}$ & ${ }_{78} \mathrm{Pt}^{195}$ & $82^{2 p^{206}}$ & $90^{\mathrm{Th}^{232}}$ & $92^{2} \mathrm{U}^{235}$ \\
\hline $\begin{array}{l}2 \\
3\end{array}$ & & & & & & & & & \\
\hline $\begin{array}{l}4 \\
6\end{array}$ & & & & & & & & & \\
\hline $\begin{array}{l}7 \\
8\end{array}$ & 0.13 & 0.021 & & 0.00001 & & & & & \\
\hline $\begin{array}{r}9 \\
10\end{array}$ & 8.03 & 1.84 & 0.34 & 0.0086 & & & & & \\
\hline $\begin{array}{l}11 \\
12\end{array}$ & 109 & 39.2 & 11.0 & 0.491 & 0.026 & & & & 0.000024 \\
\hline $\begin{array}{l}13 \\
14\end{array}$ & 384 & 238 & 111 & 10.1 & 0.818 & 0.10 & 0.036 & 0.0048 & 0.0026 \\
\hline $\begin{array}{l}16 \\
18\end{array}$ & $\begin{array}{l}663 \\
885\end{array}$ & $\begin{array}{l}525 \\
773\end{array}$ & $\begin{array}{l}371 \\
646\end{array}$ & $\begin{array}{l}94.0 \\
324\end{array}$ & $\begin{array}{l}11.8 \\
93.0\end{array}$ & $\begin{array}{l}1.96 \\
20.4\end{array}$ & $\begin{array}{l}0.79 \\
8.75\end{array}$ & $\begin{array}{l}0.148 \\
1.62\end{array}$ & $\begin{array}{l}0.091 \\
0.966\end{array}$ \\
\hline $\begin{array}{l}20 \\
22\end{array}$ & 1199 & $\begin{array}{l}971 \\
1130\end{array}$ & $\begin{array}{l}873 \\
1058\end{array}$ & $\begin{array}{l}590 \\
822\end{array}$ & $\begin{array}{l}310 \\
570\end{array}$ & $\begin{array}{l}124 \\
350\end{array}$ & $\begin{array}{l}67.7 \\
249\end{array}$ & $\begin{array}{l}18.0 \\
106\end{array}$ & $\begin{array}{l}11.7 \\
76.8\end{array}$ \\
\hline $\begin{array}{l}24 \\
26\end{array}$ & 1405 & 1365 & 1333 & $\begin{array}{l}1015 \\
1176\end{array}$ & $\begin{array}{l}804 \\
1003\end{array}$ & $\begin{array}{l}602 \\
829\end{array}$ & $\begin{array}{l}498 \\
736\end{array}$ & $\begin{array}{l}312 \\
561\end{array}$ & $\begin{array}{l}257 \\
501\end{array}$ \\
\hline $\begin{array}{l}28 \\
30\end{array}$ & 1547 & 1528 & 1526 & 1427 & 1315 & 1191 & 1123 & 996 & 945 \\
\hline $\begin{array}{l}34 \\
38\end{array}$ & $\begin{array}{l}1649 \\
1724\end{array}$ & $\begin{array}{l}1647 \\
1735\end{array}$ & $\begin{array}{l}1666 \\
1772\end{array}$ & $\begin{array}{l}1611 \\
1752\end{array}$ & $\begin{array}{l}1547 \\
1724\end{array}$ & $\begin{array}{l}1461 \\
1669\end{array}$ & $\begin{array}{l}1413 \\
1636\end{array}$ & $\begin{array}{l}1328 \\
1584\end{array}$ & $\begin{array}{l}1286 \\
1550\end{array}$ \\
\hline $\begin{array}{l}42 \\
46\end{array}$ & $\begin{array}{l}1782 \\
1826\end{array}$ & $\begin{array}{l}1802 \\
1855\end{array}$ & $\begin{array}{l}1854 \\
1918\end{array}$ & $\begin{array}{l}1861 \\
1948\end{array}$ & $\begin{array}{l}1864 \\
1975\end{array}$ & $\begin{array}{l}1832 \\
1964\end{array}$ & $\begin{array}{l}1812 \\
1953\end{array}$ & $\begin{array}{l}1787 \\
1950\end{array}$ & $\begin{array}{l}1759 \\
1928\end{array}$ \\
\hline
\end{tabular}


iable [I]

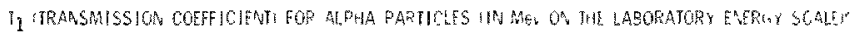

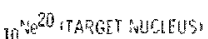

\begin{tabular}{|c|c|c|c|c|c|c|c|c|c|c|c|c|c|c|}
\hline 2 & 2 & 3 & 4 & 8 & 7 & 8 & 10 & 14 & 16 & 20 & 24 & 30 & 35 & $t^{4} 6$ \\
\hline 0 & 7704 & 7582 & 5551 & 9403 & 9521 & $039 \mathrm{I}$ & 9451 & $400]$ & $04 \pi \underline{I}$ & 0481 & $9.44]$ & 9301 & 4241 & (1) 141 \\
\hline 1 & 4.604 & 3.422 & 259] & 8.001 & 0,043 & $9.48 !$ & 0.741 & 9.671 & 0.01 & 0.481 & 0.411 & 0.331 & 9.241 & 2,14] \\
\hline 2 & 1.324 & 1,752 & 2,411 & 8.541 & 9.011 & 9.141 & $2,20 \mathrm{~g}$ & 0.401 & 0.421 & $7,4]$ & 9.431 & ६.3nt & 9.21 & 0.121 \\
\hline 3 & 2.455 & 2.953 & 4.342 & $5.35]$ & 7.941 & 9.251 & 9.891 & 9.681 & 9.561 & 0.421 & 9.361 & 9,301 & $9.2 \mathrm{II}$ & 9.10! \\
\hline 4 & 2.986 & 0.484 & 1.608 & 3.542 & 5601 & 7.032 & 8.371 & $9.21]$ & $9.43 \mathrm{~L}$ & 9. 471 & 9.401 & 0.291 & 9.171 & 9.681 \\
\hline 5 & & 4755 & 1.183 & $0.20 ?$ & $2.28 \mathrm{I}$ & $5.40 !$ & 9.621 & 9.481 & 9.521 & 0.231 & 9.271 & 9.241 & 9.151 & 9.031 \\
\hline 6 & & 6.356 & 2.144 & 1,392 & $0,61 ?$ & $1,19 \underline{\underline{L}}$ & 4,131 & $9.17 \mathrm{~g}$ & $9.67 !$ & $9.57 \div$ & $9.34]$ & $0.18 \mathrm{l}$ & $90 \mathrm{By}$ & $9.00 \mathrm{I}$ \\
\hline 7 & & & 1.4 .45 & 1.283 & 0,693 & 2842 & $2.45 ?$ & 1013 & $7.9 \mathrm{n}$ & 8.921 & 9.201 & 9.221 & 9,051 & 5.031 \\
\hline 8 & & & 1.896 & 1,524 & 6.944 & 2.473 & 2004 & $4.92 \mathrm{l}$ & 9,031 & 9.181 & 8.861 & 8.671 & 8.4.41 & 8.891 \\
\hline 9 & & & & 2.50 & 1.251 & 4.844 & 4.213 & 7.248 & 201 & 7.831 & $283 \mathrm{I}$ & 4.101 & $893]$ & 8.701 \\
\hline 10 & & & & 4.160 & 2.145 & 7.935 & 0.324 & $1,70^{\circ}$ & 7.502 & $3.20 \mathrm{~g}$ & 6.341 & 0.163 & 3.931 & 8,701 \\
\hline 11 & & & & & 4.28 & 1.735 & 1.45 & 2,053 & 8.293 & $8.1 k_{2}^{\circ}$ & 5.619 & $7.19 \mathrm{l}$ & $86 \times 1$ & 8,751 \\
\hline 12 & & & & & & $2 \% 0$ & 3,65 & 1.304 & 2.15 & 1,112 & $0.5 \%$ & 9.071 & $x_{n} 01$ & $8.38 \mathrm{I}$ \\
\hline 13 & & & & & & $1 M 6$ & 9.60 & 2.214 & 0.684 & 2,703 & 1.402 & 1,031 & 0.201 & 8.531 \\
\hline 14 & & & & & & & $5 E_{0 y}$ & $65^{2}$ & 2.393 & 0.754 & 4.613 & 2.65 & 2301 & 877 \\
\hline 15 & & & & & & & & 2.115 & 0.375 & 1.566 & 1,123 & 8.993 & 5672 & 2.601 \\
\hline 10 & & & & & & & & $0.57 \mathrm{t}$ & $18^{385}$ & 3.275 & 2074 & 3.003 & 2.152 & 8.502 \\
\hline 17 & & & & & & & & & & 6.100 & 7.015 & 9.374 & 859 & 3,522 \\
\hline 18 & & & & & & & & & & 1.050 & 1.495 & $2.66 C_{6}$ & 3.253 & 1.572 \\
\hline 19 & & & & & & & & & & & $2.8 \%$ & 0.775 & 1.143 & 0.803 \\
\hline 20 & & & & & & & & & & & & 1.555 & 3.614 & 2.833 \\
\hline 21 & & & & & & & & & & & & 3.280 & 1.639 & 1,403 \\
\hline 22 & & & & & & & & & & & & & 265 & 2,564 \\
\hline 23 & & & & & & & & & & & & & 6.156 & 1,034 \\
\hline 24 & & & & & & & & & & & & & 1.316 & 2005 \\
\hline $\begin{array}{l}25 \\
26\end{array}$ & & & & & & & & & & & & & & $\begin{array}{l}7.5200 \\
1.720\end{array}$ \\
\hline
\end{tabular}

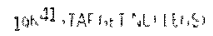

\begin{tabular}{|c|c|c|c|c|c|c|c|c|c|c|c|c|c|c|}
\hline 2 & 4 & b & है & 10 & 12 & $3 \hat{3}$ & 15 & $m$ & 20 & 30 & 32 & $x$ & $a^{2}$ & 40 \\
\hline 0 & 1.393 & 2.451 & 8.671 & $9.8 \% 1$ & $0.5]$ & 5,9, & $9.40 ?$ & 9,971 & 0,931 & 9.971 & 9,901 & $0.95 !$ & 5047 & 4.031 \\
\hline 1 & 3.043 & 201 & 8,041 & $957 !$ & 1,364 & $9.5 \mathrm{Ml}$ & 9.81 & 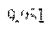 & 8.971 & $S_{0}\left(4_{2}\right]$ & 7.901 & 3.953 & 50.11 & 943 \\
\hline 2 & 4.814 & 1.221 & 7.651 & 9.041 & 9.291 & 9931 & 901 & 9.071 & 0.071 & 9.01 & 0.051 & 0.041 & 0.41 & 0901 \\
\hline 3 & 1.860 & 5.862 & $5.37 \mathrm{I}$ & \& $]$ & 0.831 & 0,53 & 9.891 & 9.977 & Q. $\log \frac{l}{3}$ & $9 . \sin 1$ & $0.95]$ & 2.951 & $803]$ & 9,033 \\
\hline 4 & 4.755 & 1.712 & 3.801 & 8,571 & $9.59 \mathrm{~g}$ & 3.821 & $9,95 !$ & 9.981 & 9.71 & 9.901 & 9.531 & 0,41 & 9.091 & 7.723 \\
\hline 5 & 1.145 & 5,113 & 1.461 & 6,631 & $0.3 \leq$ & Q & $9,40 !$ & $4,4]$, & 0,951 & $9.02]$ & $985]$ & 0.31 & 0.931 & 95 \\
\hline 6 & 2,200 & 1.133 & $475 ?$ & 3.831 & ?.Y9? & 0,331 & 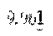 & $9,9 \mathrm{~m}$ & 9.601 & 9.91 & 9.041 & 8.631 & $y 21$ & 4612 \\
\hline$?$ & & 2.13. & 0.493 & 1.311 & 5.731 & 9.771 & $9,7 \wedge 1$ & $0,9 \mathrm{gl}$ & 0.001 & 9,051 & $9.94 !$ & 4.021 & 9.911 & $n$ an! \\
\hline$\not$ & & $\therefore, 02$ & 2.033 & 2.792 & $20 \mathrm{~m}$ & of 1 & 9.821 & 9.931 & 9.801 & Q.4? & 0.931 & 9.921 & 9,911 & 0,601 \\
\hline 9 & & Q.LItg & $4.15 \%$ & 4,572 & $5.70 ?$ & 2041 & 8472 & 9,001 & 0.911 & 9.031 & 9,901 & 0.001 & 9.89 & $180\}$ \\
\hline 10 & & 1.99 & +58 & 1.323 & 1.232 & $093^{\circ}$ & $5,31 k$ & $0.12]$ & 0,891 & $9 x^{2}+4^{2}$ & 0.5321 & 4,007 & $7 \times 81$ & $9, M_{1}$ \\
\hline 11 & & & 2415 & 3524 & 2,053 & $1.44^{3}$ & 230 & 841 & 0.591 & 9.74 & 9.31 & 0.891 & a.ne! & $3.80 \mathrm{l}$ \\
\hline 12 & & & 6.146 & 9.775 & 1.474 & 3.862 & 5.592 & 4.015 & 0.151 & 9.921 & 9.811 & 9,801 & 9.831 & 9.841 \\
\hline 13 & & & $1.50 \mathrm{~g}$ & 232 & 2.254 & 1.143 & $1,4 \sqrt{4} Z$ & 231 & $5.2 \mathrm{~m}$ & 0.001 & $9.9 \leq 1$ & 0.021 & 4,821 & 9.701 \\
\hline 14 & & & & 8.100 & 7.145 & 3.784 & 4083 & 3,312 & $2[9]$ & $6.18 !$ & 8601 & 0.731 & $0,9 ! 1$ & 9.851 \\
\hline 15 & & & & 2,33 & 2.275 & 1.290 & $1.2 / 3$ & 1.012 & 5.252 & $2,5\}$ & 7.19! & 8.701 & 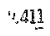 & 9.75 \\
\hline 16 & & & & & 3.060 & 4,4055 & 3.864 & 3.503 & $1.75 ?$ & 6.712 & 2621 & $7.44 !$ & g. IgI & 4.321 \\
\hline 17 & & & & & & 1.515 & 1,094 & 1.203 & 6.823 & 2.442 & 7.522 & 2.331 & 6.431 & 9.011 \\
\hline 18 & & & & & & $5.0 \%$ & 2835 & 4.194 & 2093 & 1022 & 2.79 & 7.772 & 2001 & 4,031 \\
\hline 19 & & & & & & & 6.590 & 1,284 & 1.013 & 4.403 & 1.32 & 3.302 & 3.002 & 1701 \\
\hline 20 & & & & & & & 1.406 & 3.585 & 3.534 & $1.6^{n} 5$ & 0.073 & $1.55 ?$ & $345 ?$ & 7.182 \\
\hline 21 & & & & & & & & 9.140 & 1,134 & 7.014 & 2.713 & 7.683 & 1.692 & 3.442 \\
\hline 22 & & & & & & & & 2126 & 3.315 & 2.53 & 1.153 & 3.553 & 8,503 & 1752 \\
\hline 23 & & & & & & & & & 8.846 & $829 ?$ & 4484 & 1.603 & 4.233 & 9.133 \\
\hline 24 & & & & & & & & & & 2485 & 1.614 & 0.764 & 2.023 & 4.733 \\
\hline 25 & & & & & & & & & & 6.806 & 5,315 & $2,6.94$ & 9004 & 2.373 \\
\hline 26 & & & & & & & & & & 1.736 & 1.605 & 9.355 & 3.754 & 1. 123 \\
\hline 27 & & & & & & & & & & & & 3.055 & 1.434 & 4814 \\
\hline 28 & & & & & & & & & & & & 7.150 & 4975 & 3.964 \\
\hline 29 & & & & & & & & & & & & & 1,505 & 7.225 \\
\hline 30 & & & & & & & & & & & & & 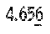 & 2.4 .45 \\
\hline 31 & & & & & & & & & & & & & 1.906 & 7.616 \\
\hline
\end{tabular}


Tate III (Conted.)

20TU $48_{\text {TTARGRT NUCLEUS }}$

\begin{tabular}{|c|c|c|c|c|c|c|c|c|c|c|}
\hline$\ell$ & 4 & 6 & 7 & 8 & 9 & 10 & 11 & 12 & 13 & 14 \\
\hline 0 & 1.074 & 6.612 & 3.321 & 0.891 & 8.791 & 9.501 & 9.761 & 9.801 & 9.911 & 9.941 \\
\hline 1 & 8005 & 5.102 & 2.711 & 6.151 & 8.371 & 9.331 & 9.221 & 9.801 & 9.961 & 9.991 \\
\hline 2 & 4055 & 3002 & 1.911 & 5.301 & 7.361 & 9.131 & $9.58 \mathrm{I}$ & $9.77 \underline{1}$ & $0.86 ?$ & 9.911 \\
\hline 3 & 1.645 & 1.352 & 9.692 & 3.461 & 6.581 & 8.531 & $9.4 \mathrm{II}$ & 9.761 & 9.901 & 9.961 \\
\hline 4 & 4.996 & 4.223 & 40.042 & 1.831 & 4.741 & 7.301 & 8.691 & 9.341 & 0.651 & 9.811 \\
\hline 5 & 1.316 & 1.303 & 1.192 & 6.602 & 2.34 & 5.161 & 7,621 & 8.951 & 9.521 & 9.761 \\
\hline 6 & & 3224 & 3.183 & 1.972 & 8.342 & 2421 & 4.831 & 7.071 & 8.511 & 9.291 \\
\hline 7 & & 7.045 & 6.954 & 4.533 & 2.192 & 8.122 & 2.861 & 4.531 & 6.701 & 8.131 \\
\hline 8 & & 1.625 & 1.594 & 1.023 & 4.873 & 1.872 & 5.992 & 1.601 & 3.431 & 5.721 \\
\hline 9 & & 3.780 & 3.755 & 2.394 & 1.153 & 4.473 & 1.442 & $42 \%$ & 1,061 & 2.253 \\
\hline 10 & & & 9.176 & 5.985 & 2.774 & 1.043 & 3.383 & 9.893 & 2.672 & 6.592 \\
\hline 11 & & & 2.336 & 1.55 & 7,502 & 2804 & $8.7 ? 4$ & 2.423 & 6.133 & 1.462 \\
\hline 12 & & & & 4.146 & 2.055 & 8.095 & 2.574 & 7.054 & $1.74 \underline{3}$ & 3.983 \\
\hline 13 & & & & 1.076 & 5.886 & 2.395 & 7.845 & 2.194 & 5.434 & 1.233 \\
\hline 14 & & & & & 1.608 & 7.035 & 2.435 & 7,5 & 1.704 & 4.104 \\
\hline 15 & & & & & & 2.036 & $7.4 \%$ & 2.285 & 0.025 & 1.424 \\
\hline 10 & & & & & & & 2.216 & 7.286 & 2005 & 4.925 \\
\hline 17 & & & & & & & & & 0.750 & 1.715 \\
\hline 18 & & & & & & & & & & 5,780 \\
\hline
\end{tabular}

$23^{51 / T A P G E T ~ N U C L E S ~}$

\begin{tabular}{|c|c|c|c|c|c|c|c|c|c|c|c|c|c|c|c|c|c|c|}
\hline E & 4 & 6 & 8 & 10 & 12 & 14 & 16 & 18 & 20 & 32 & 24 & 20 & 88 & 30 & 34 & 38 & 42 & 40 \\
\hline 0 & 4.725 & 4.132 & $6.11 \mathrm{I}$ & 9.34 & 9.831 & 9.941 & 0.981 & 10001 & 10.001 & 1000 & 10001 & 990 & 3.301 & 0.991 & 9,091 & 9.981 & 9.931 & 9.481 \\
\hline i & 3.525 & 3.62 & $5.41 !$ & 9.211 & 9.871 & 9.981 & 9.941 & $0.98]$ & $70 \%$ & 9.91 & 909 & $0,09 \mathrm{~s}$ & $9.69 !$ & 9041 & 9.991 & 9.981 & $9.69 !$ & 4.081 \\
\hline 2 & 1.855 & $1.87 ?$ & 4.471 & 8.891 & 9.741 & 9.921 & 9.971 & 9.991 & 10.001 & 10.001 & $10.00 \%$ & 9.961 & 9.911 & 9991 & 9.901 & 9.982 & \& 981 & 9.981 \\
\hline 3 & 7.756 & 8.373 & 2.821 & 8.271 & 9.711 & 9.941 & 9.071 & 9.981 & 0.481 & Q.901 & 4.941 & $9.0 \mathrm{dg}$ & 9.991 & 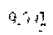 & 0,090 & 9.081 & 9,981 & 9,091 \\
\hline 4 & 2.406 & 3023 & $1.45 ?$ & 6.841 & 9.271 & 9.821 & 0.961 & 9.991 & $10.00 \mathrm{~s}$ & $10, \mathrm{ng}]$ & $0,04:$ & 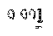 & 9001 & 9,091 & 9,931 & 9.981 & 9.981 & 90 \\
\hline 5 & & 8.564 & 5.142 & 4.691 & 8.751 & 9.601 & 9.881 & 9.941 & 0,971 & 9.981 & 0,001 & 0,091 & 9.991 & 9.991. & 9.981 & 9.981 & 9.951 & 991 \\
\hline 4 & & 2.214 & 1522 & 2.111 & 6.871 & $9,28 \mathrm{Z}$ & 0,861 & 9,971 & 0.681 & 0,081 & 4.081 & 9.481 & 9991 & 0,081 & 9.381 & 9.981 & 9.981 & 9.91 \\
\hline 7 & & 5.125 & 3.693 & 1.072 & 4.211 & 7.081 & 9.381 & 9.811 & 9,951 & 9,901 & 00.001 & 9.991 & 9.991 & 9.981 & $9.98 \mathrm{~m}$ & $9.97 !$ & 9.972 & $9.97 !$ \\
\hline 9 & & 1.215 & 854 & 1.692 & 1.532 & 5.031 & 8.691 & 0.571 & 9,811 & 0,91 & 0,012 & $0,07 \mathrm{~g}$ & 0.081 & 9.991 & $4.98 ?$ & 9.971 & $9.07 !$ & 0,961 \\
\hline 9 & & 2.930 & 2.084 & 4103 & 4902 & 2.231 & 0.032 & 8.891 & 9.721 & 9.92 & จ. 951 & 6,931 & 9.951 & 4.961 & $3.9 !$ & $9.97 !$ & 9.971 & 9.901 \\
\hline 10 & & & 5,295 & 9.984 & 908 & 6652 & 2.751 & $0.09 \mathrm{l}$ & 8,451 & 9,511 & $9.88 ?$ & 0,091 & 10001 & 9991 & $9.96 \mathrm{il}$ & १.961 & 9.561 & 9.961 \\
\hline 11 & & & 1.425 & 2.724 & 2.473 & 1.552 & 7.832 & $2.90 \mathrm{t}$ & o 201 & 8331 & 9.251 & 54,602 & 5861 & 9451 & 0.991 & 9.921 & 9.051 & $9, ., 41$ \\
\hline 12 & & & $381 \mathrm{~b}$ & 7.975 & 7.254 & 4.223 & 1,922 & 7.512 & $2.46 !$ & $5.6 \mathrm{ll}$ & 8.291 & 9.321 & 9.631 & $9.76 \mathrm{I}$ & $9.90 \mathrm{l}$ & 9.951 & 9.961 & 9.951 \\
\hline 13 & & & 106 & $\therefore 365$ & 2.384 & 1.323 & $5.6 \%$ & 2.082 & 0762 & 1.911 & $4,30]$ & 7.231 & 9.121 & 9.751 & $3.88 \mathrm{~g}$ & $9.8 \mathrm{I}$ & 9.911 & 9.931 \\
\hline 14 & & & & 7.150 & 7.375 & 4.924 & 1883 & 6.453 & 1.992 & 5.752 & 1.511 & 3,291 & 5711 & 7.911 & 9.831 & 9.981 & $9.91 \underline{1}$ & $9.89 \mathrm{~s}$ \\
\hline 15 & & & & 2,156 & 2.405 & $1.5 \mathrm{MA}$ & 6.724 & 2.293 & 6603 & 1.732 & 4.382 & 301 & $2.49 !$ & 4561 & 8.13I & 0,611 & 9,001 & 0.581 \\
\hline Is & & & & & 7.726 & 5.385 & 2.484 & 8.098 & 2.523 & 0.273 & 1,472 & $3.2 \mathrm{o}_{2}^{3}$ & 7.132 & 1,571 & 5.531 & 8.381 & 0.46 .1 & $9.97]$ \\
\hline 17 & & & & & & & 9.195 & 3.334 & 1013 & 2.443 & 5,003 & 1.252 & 2.492 & 4.852 & 1.861 & 5.921 & 8.791 & $0.38]$ \\
\hline 18 & & & & & & & 3,453 & $1.2 \%$ & 4.65 .4 & 9.320 & 2.503 & 5.293 & 1.042 & $1.94 \%$ & 6.182 & 1,441 & 5.571 & 9,081 \\
\hline 19 & & & & & & & & 4.655 & 1.624 & 3.384 & 1.093 & 2.33 & 4.563 & 8.603 & $2.60 \%$ & $7.08 ?$ & 1.881 & 4.781 \\
\hline 20 & & & & & & & & 1.62 & 6.275 & 1.154 & 4,73 & 1.053 & 2.600 & 3.923 & $1,20 \%$ & 3.132 & 7,532 & 2.751 \\
\hline 21 & & & & & & & & 5.376 & 2.335 & 3.585 & 2074 & 4.704 & 8.434 & 1.763 & 5.763 & 1.512 & 3.502 & $7.60 ?$ \\
\hline 22 & & & & & & & & & & & 9.075 & 2.104 & 3.354 & 7.584 & 2.763 & 7.513 & 1.752 & 3.702 \\
\hline 23 & & & & & & & & & & & 3.955 & 0,195 & 1.24 & 30.9 & 1.293 & 3.783 & 8.983 & 1.912 \\
\hline 24 & & & & & & & & & & & & & & 1.164 & 5.11. & 1.873 & 4.693 & 1.012 \\
\hline 25 & & & & & & & & & & & & & & 4.015 & 2.34 & 8.994 & 2.43 & 5.433 \\
\hline 26 & & & & & & & & & & & & & & 1,285 & 9.115 & 4.004 & 1.223 & 2523 \\
\hline 27 & & & & & & & & & & & & & & & & & 5.874 & 1.543 \\
\hline 28 & & & & & & & & & & & & & & & & & & 7.774 \\
\hline 29 & & & & & & & & & & & & & & & & & & 3.714 \\
\hline 30 & & & & & & & & & & & & & & & & & & 1.64 \\
\hline 31 & & & & & & & & & & & & & & & & & & 0.765 \\
\hline
\end{tabular}


Table III (Cont'd.)

$24 \mathrm{Cr}^{52}$ (TARGET NUCLEUS)

\begin{tabular}{|c|c|c|c|c|c|c|c|c|c|c|}
\hline$\ell$ & 4 & 6 & 7 & 8 & 9 & 10 & 11 & 12 & 13 & 14 \\
\hline 0 & 1.725 & 2.182 & 1.621 & 5.011 & 7.861 & 9.121 & 9.601 & 9.791 & 9.881 & 9.931 \\
\hline 1 & 1.295 & 1.672 & 1.291 & 4.311 & 7.361 & 8.931 & 9.571 & $9.82 \underline{I}$ & 9.931 & 9.971 \\
\hline 2 & 6.786 & 9.763 & 8.462 & 3.391 & 6.651 & 8.541 & 9.341 & $9.67 I$ & 9.821 & 9.901 \\
\hline 3 & 2.866 & 4.413 & 4.122 & 1.991 & $5.06 \underline{1}$ & 7.711 & 9.051 & $9.61]$ & 9.831 & 9.921 \\
\hline 4 & $9.54 \underline{7}$ & 1.593 & 1.662 & 9.692 & 3.191 & 6.081 & 8.081 & 9.081 & 9.551 & $9.77 \underline{1}$ \\
\hline 5 & & 4.634 & 5.043 & 3.302 & 1.411 & 3.821 & 6.591 & 8.401 & 9.251 & 9.621 \\
\hline 6 & & 1.234 & 1.393 & 9.763 & 4.692 & 1.591 & 3.751 & 6.241 & $8.07 \underline{1}$ & 9.091 \\
\hline 7 & & 2.955 & 3.334 & 2.403 & 1.262 & 5.082 & $1.57 \underline{1}$ & 3.541 & 5.831 & 7.601 \\
\hline 8 & & 7.236 & 8.155 & 5.734 & 2.953 & $1.21 ?$ & 4.162 & 1.201 & 2.781 & 5.021 \\
\hline 9 & & 1.786 & 2.045 & 1.484 & 7.364 & 3.023 & 1.052 & 3.152 & 8.232 & 1.861 \\
\hline 10 & & & 5.196 & 3.755 & $1.89 \underline{4}$ & 7.544 & $2.56 \underline{3}$ & 7.743 & 2.142 & 5.402 \\
\hline 11 & & & 1.336 & 1.025 & 5.295 & $2.10 \mathrm{~A}$ & 6.914 & 1.993 & 5.203 & 1.282 \\
\hline 12 & & & & 2.746 & 1.515 & 6.225 & 2.084 & 5.944 & 1.523 & 3.553 \\
\hline 13 & & & & & 4.266 & 1.865 & 6.475 & 1.894 & 4.844 & 1.123 \\
\hline 14 & & & & & 1.186 & 5.606 & 2.035 & 6.155 & 1.624 & 3.814 \\
\hline 15 & & & & & & 1.606 & 6.286 & 2.005 & 5.485 & 1.334 \\
\hline 16 & & & & & & & 1.886 & 6.446 & 1.865 & 4.665 \\
\hline 17 & & & & & & & & & 6.146 & 1.635 \\
\hline 18 & & & & & & & & & & 5.596 \\
\hline
\end{tabular}

$25 \mathrm{Mn}$ 33(TARGET NUCLEUS)

\begin{tabular}{|c|c|c|c|c|c|c|c|c|c|c|}
\hline$\ell$ & 4 & 6 & 7 & 8 & 9 & 10 & 11 & 12 & 13 & 14 \\
\hline 0 & 6.306 & 1.122 & 1.001 & 3.871 & 7.131 & 8.811 & 9.481 & 9.741 & 9.861 & 9.921 \\
\hline 1 & 4.756 & 8.683 & $7.88 ?$ & $3.25]$ & 6.541 & 8.551 & 9.411 & 9.761 & 9.901 & 9.961 \\
\hline 2 & 2.596 & 5.033 & 5.032 & 2.441 & 5.721 & 8.081 & 9.151 & 9.591 & 9.781 & 9.881 \\
\hline 3 & 1.166 & 2.303 & 2.432 & 1.361 & $4.07 \underline{]}$ & 7.021 & 8.731 & 9.471 & 9.771 & 9.001 \\
\hline 4 & $3.43 i$ & 8.384 & $9.67 \underline{3}$ & 6.292 & 2.391 & 5.241 & 7.571 & 8.841 & $9.43 I$ & $9.72 \underline{1}$ \\
\hline 5 & & 2.514 & 2.983 & 2.112 & 9.832 & 3.001 & 5.821 & 7.951 & 9.041 & 9.531 \\
\hline 6 & & 6.865 & 8.374 & 6.253 & 3.212 & 1.181 & 3.051 & 5.551 & 7.621 & 8.851 \\
\hline 7 & & 1.725 & 2.084 & $1.57 \underline{3}$ & 8.573 & 3.622 & 1.191 & 2.911 & 5.201 & $7.16 \mathrm{I}$ \\
\hline 8 & & $4.37 \underline{6}$ & $5.27 \underline{5}$ & 3.894 & 2083 & $8.80 \underline{3}$ & 3.112 & 9.272 & 2.281 & 4.391 \\
\hline 9 & & 1.106 & 1.365 & 1.014 & 5.334 & 2.243 & 7.933 & 2.442 & $6.57 ?$ & 1.541 \\
\hline 10 & & & $3.55 \underline{6}$ & 2.695 & 1.414 & 5.784 & 2.003 & 6.123 & 1.712 & 4.392 \\
\hline 11 & & & & 7.466 & 4.015 & 1.654 & 5.554 & 1.623 & 4.303 & 1.062 \\
\hline 12 & & & & 2.006 & 1.165 & 4.945 & 1.694 & 4.944 & 1.283 & 3.033 \\
\hline 13 & & & & & 3.260 & 1.495 & 5.315 & 1.594 & 4.144 & 9.754 \\
\hline 14 & & & & & & 4.526 & 1.685 & 5.195 & 1.394 & 3.334 \\
\hline 15 & & & & & & 1.266 & $5.17 \underline{\underline{6}}$ & 1.705 & 4.755 & 1.174 \\
\hline 16 & & & & & & & $1.57 \underline{6}$ & 5.456 & $1.61 \underline{5}$ & 4.115 \\
\hline 17 & & & & & & & & & 5.306 & $1.44 \underline{5}$ \\
\hline 18 & & & & & & & & & & 4.936 \\
\hline
\end{tabular}


Table III (Cont'd.)

$25 \mathrm{Mn}^{55}$ (TARGET NUCLEUS)

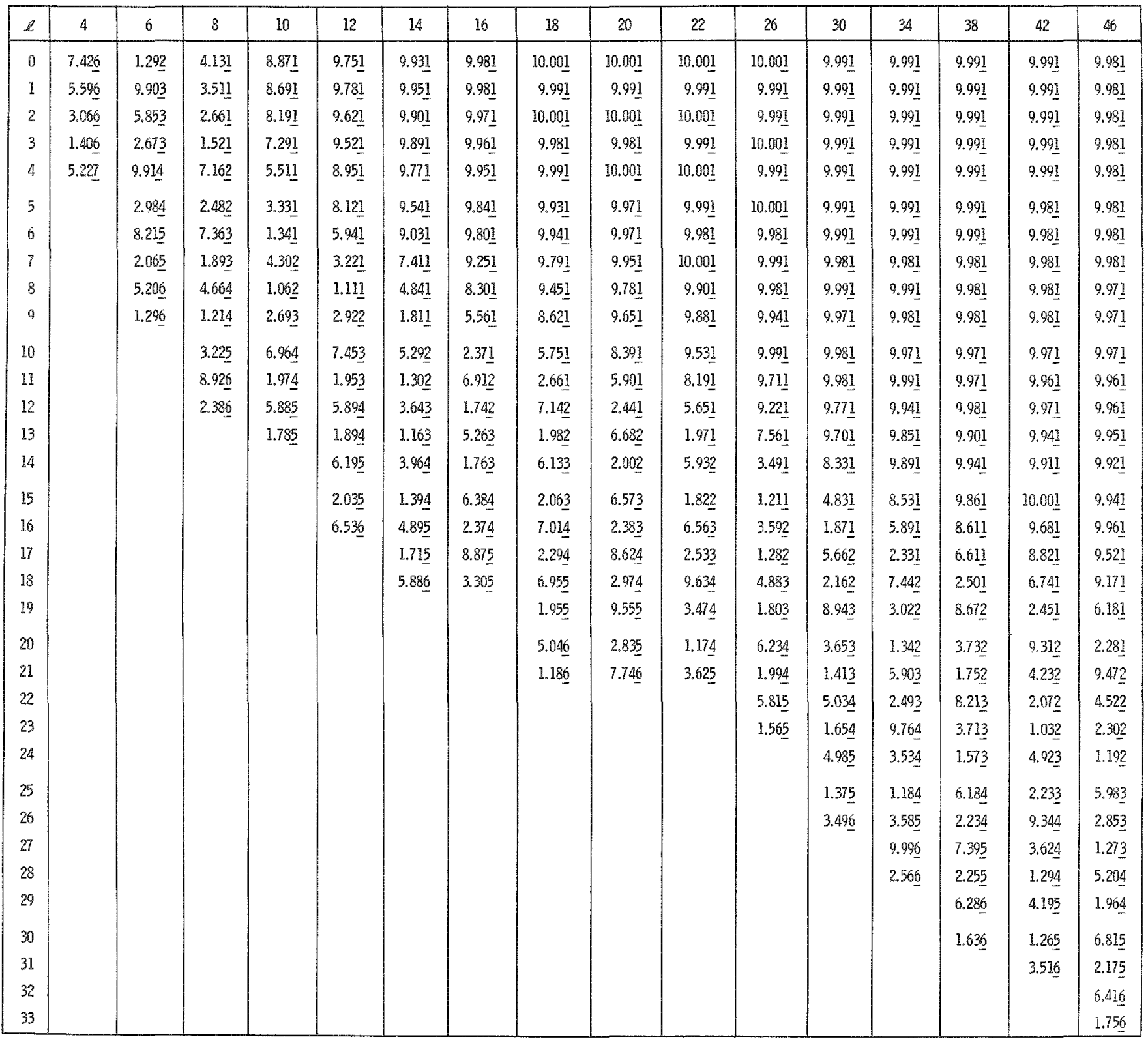


Table III iCont'd.) ${ }_{26} \mathrm{Fe}^{56}$ (TARGET NUCLEUS)

\begin{tabular}{|c|c|c|c|c|c|c|c|c|c|c|}
\hline$\ell$ & 4 & 6 & 7 & 8 & 9 & 10 & 11 & 12 & 13 & 14 \\
\hline 0 & $2.67 \underline{6}$ & 6.563 & 6.692 & 3.051 & 6.451 & 8.501 & $9.35 \underline{\underline{l}}$ & 9.691 & $9.84]$ & 9.911 \\
\hline 1 & 2.046 & 5.053 & 5.232 & $2.53]$ & 5.881 & $8.24 \underline{1}$ & 9.201 & 9.711 & $9.87 \underline{1}$ & $9.94 \underline{1}$ \\
\hline 2 & 1.246 & 2.983 & 3.332 & 1.841 & 4.971 & $7.64 \underline{\underline{I}}$ & 8.951 & 9.511 & $9.76 \underline{1}$ & $9.87 \underline{I}$ \\
\hline 3 & 4.367 & 1.383 & 1.612 & $1.00 \mathrm{I}$ & 3.421 & 6.511 & 8.481 & 9.361 & $9.72 \underline{1}$ & 9.861 \\
\hline 4 & & 5.174 & 6.493 & 4.572 & 1.911 & 4.641 & 7.191 & 8.671 & 9.371 & 9.701 \\
\hline 5 & & 1.604 & $2.06 \underline{3}$ & $1.50 ?$ & 7.792 & $2.56]$ & $5.34]$ & 7.631 & 8.871 & 9.441 \\
\hline 6 & & 4.565 & 5.944 & 4.683 & 2.522 & 9.782 & 2.701 & 5.221 & 7.431 & 8.771 \\
\hline 7 & & 1.195 & 1.544 & 1.223 & 6.963 & 3.042 & 1.031 & 2.621 & 4.881 & 6.941 \\
\hline 8 & & 3.106 & 4.035 & 3.134 & $1.74 \underline{3}$ & 7.613 & 2.782 & 8.522 & 2.141 & 4.211 \\
\hline 9 & & & 1.075 & 8.345 & 4.584 & 1.983 & 7.153 & 2.252 & 6.192 & 1.481 \\
\hline 10 & & & 2.846 & $2.27 \underline{5}$ & 1.254 & 5.274 & 1.863 & 5.833 & 1.652 & 4.272 \\
\hline 11 & & & & 6.326 & 3.595 & 1.524 & 5.264 & 1.583 & 4.263 & $1.07 ?$ \\
\hline 12 & & & & 1.706 & $1.04 \underline{5}$ & 4.615 & 1.624 & 4.834 & 1.283 & $3.07 \underline{3}$ \\
\hline 13 & & & & & 3.036 & 1.415 & 5.135 & 1.574 & 4.164 & 9.964 \\
\hline 14 & & & & & & 4.176 & 1.635 & 5.175 & 1.414 & 3.424 \\
\hline 15 & & & & & & 1.226 & 5.016 & 1.095 & 4.835 & 1.214 \\
\hline 16 & & & & & & & 1.546 & 5.506 & 1.645 & 4.275 \\
\hline 17 & & & & & & & & & 5.466 & 1.405 \\
\hline 18 & & & & & & & & & & 5.116 \\
\hline
\end{tabular}

${ }_{27} \mathrm{COO}^{55}$ itarget nucleus?

\begin{tabular}{|c|c|c|c|c|c|c|c|c|c|c|}
\hline$\ell$ & 4 & 6 & 7 & 8 & 9 & 10 & 11 & 12 & 13 & 14 \\
\hline 0 & 8.057 & 2.853 & 3.402 & 1.941 & 5.221 & 7.871 & 9.101 & 9.581 & 9.791 & 9.881 \\
\hline 1 & $6.50 ?$ & 2.223 & 2.682 & 1.581 & 4.581 & $7.44]$ & 8.931 & $9.55]$ & $9.81 \underline{1}$ & 9.921 \\
\hline 2 & 3.287 & 1.303 & 1.652 & 1.091 & 3.691 & 6.751 & 8.551 & $9.33]$ & 9.671 & 9.821 \\
\hline 3 & 1.687 & 6.104 & 8.023 & 5.682 & 2.311 & $5.31]$ & 7.791 & 9.061 & 9.601 & 9.821 \\
\hline 4 & & 2.294 & 3.173 & 2.492 & 1.201 & 3.501 & 6.271 & 8.151 & 9.111 & $9.56 \underline{1}$ \\
\hline 5 & & 7.355 & 1.023 & 8.323 & 4.522 & 1.691 & $4.14]$ & 6.771 & 8.451 & 9.251 \\
\hline 6 & & 2.155 & 3.014 & 2.533 & 1.452 & 6.102 & 1.871 & 4.101 & 6.511 & 8.221 \\
\hline 7 & & 5.866 & $8.07 \underline{5}$ & 6.704 & 3.953 & 1.802 & 6.552 & 1.851 & 3.891 & 6.111 \\
\hline 8 & & 1.586 & 2.195 & 1.794 & 1.033 & 4.603 & $1.72 ?$ & 5.462 & 1.471 & 3.191 \\
\hline 9 & & & 5.966 & 4.935 & 2.794 & 1.233 & 4.553 & 1.462 & 4.13? & 1.031 \\
\hline 10 & & & 1.576 & 1.375 & 7.845 & 3.394 & 1.223 & 3.823 & 1.092 & 2.882 \\
\hline 11 & & & & 3.886 & 2.295 & 1.014 & 3.504 & 1.083 & 2.933 & $7.36 \underline{3}$ \\
\hline 12 & & & & 1.046 & 6.686 & 3.095 & 1.124 & 3.404 & 9.084 & 2.203 \\
\hline 13 & & & & & 1.880 & 9.506 & 3.585 & 1.114 & $3.01 \underline{4}$ & 7.304 \\
\hline 14 & & & & & & 2.846 & 1.135 & 3.705 & 1.034 & 2.54 \\
\hline 15 & & & & & & & 3.096 & 1.215 & 3.535 & 8.995 \\
\hline 16 & & & & & & & 1.036 & 3.896 & 1.205 & 3.185 \\
\hline 17 & & & & & & & & & 4.016 & $1.11 \underline{5}$ \\
\hline 18 & & & & & & & & & & 3.826 \\
\hline
\end{tabular}


Table III (Cont'd.)

$32 \mathrm{Ge}^{72}$ (TARGET NUCLEUS)

\begin{tabular}{|c|c|c|c|c|c|c|c|c|c|c|c|c|c|}
\hline$\ell$ & 6 & 8 & 10 & 12 & 14 & 18 & 22 & 26 & 30 & 34 & 38 & 42 & 46 \\
\hline 0 & 1.814 & 3.492 & 4.871 & 8.971 & 9.791 & 9.991 & 10.001 & 10.001 & 10.001 & 10.001 & 10.001 & 10.001 & 10.001 \\
\hline 1 & 1.444 & 2.822 & 4.441 & 8.851 & 9.761 & 9.981 & 10.001 & 10.001 & 10.001 & 10.001 & 10.001 & 10.001 & 10.001 \\
\hline 2 & 9.145 & 1.882 & 3.56] & $8.46]$ & 9.701 & 9.981 & 10.001 & 10.001 & $10.00]$ & 10.001 & 10.001 & 10.001 & 10.001 \\
\hline 3 & 4.735 & 1.022 & 2.451 & 7.801 & 9.541 & 9.961 & 10.001 & 10.001 & $10.00 \mathrm{I}$ & 10.001 & 10.001 & 10.001 & 10.001 \\
\hline 4 & 2.075 & 4.583 & 1.361 & 6.521 & 9.261 & 9.951 & 9.991 & $10.00]$ & 10.001 & 10.001 & 10.001 & 10.001 & 10.001 \\
\hline 5 & 7.846 & 1.753 & 6.132 & 4.631 & 8.561 & 9.911 & 9.991 & 10.001 & 10.001 & 10.001 & 10.001 & 10,001 & 10.001 \\
\hline 6 & 2.706 & 5.974 & 2.232 & 2.511 & 7.281 & 9.811 & 9.981 & 10.001 & 10.001 & 10.001 & 10.001 & 10.001 & 10.001 \\
\hline 7 & 8.947 & 1.904 & $7.26 \underline{3}$ & 1.011 & 4.881 & $9.63 \underline{1}$ & 9.961 & 9.991 & 10.001 & $10.00 \mathrm{I}$ & 10.001 & $10.00 \underline{\underline{I}}$ & $10.00]$ \\
\hline 8 & & 5.865 & 2.173 & 3.322 & 2.401 & 8.961 & 9.921 & 10.001 & 10.001 & 10.001 & 10.001 & 10.001 & 10.003 \\
\hline 9 & & 1.805 & 6.514 & 9.633 & 8.272 & 7.571 & 9.741 & 9.961 & 10.001 & 10.001 & 10.001 & 9.991 & 9.991 \\
\hline 10 & & 5.426 & 2.024 & 2.843 & 2.412 & 4.691 & 9.411 & 9.911 & $9.97 \underline{1}$ & 9.991 & 10.001 & 10.001 & 9.991 \\
\hline 11 & & $1.57 \underline{6}$ & 6.395 & 8.694 & 6.793 & 1.931 & 8.041 & 9.831 & 9.981 & 9.981 & 9.991 & 9.991 & 9.991 \\
\hline 12 & & & 2.045 & 2.774 & 1.933 & 6.062 & 5.311 & 9.221 & 9.921 & 10.001 & 9.991 & 0.991 & 9.991 \\
\hline 13 & & & 6.436 & $8.86 \underline{5}$ & $5.63 \mathrm{~A}$ & 1.762 & 2.251 & 7.821 & 9.611 & 9.931 & 10.001 & 10.001 & 0.991 \\
\hline 14 & & & 1.926 & $2.75 \underline{5}$ & 1.614 & 5.633 & 6.972 & 4.611 & 9.001 & 9.781 & 9.931 & 9.981 & 9.991 \\
\hline 15 & & & & 8.1116 & 4.355 & $1.87 \underline{3}$ & 2.292 & 1.711 & 6.481 & 9.491 & 9.901 & $9.94 \underline{I}$ & 9.971 \\
\hline 16 & & & & 2.256 & I.105 & $6.17 \underline{4}$ & 8.103 & 5.762 & 3.011 & 7.541 & 9.671 & $9.97 \underline{1}$ & $9.96]$ \\
\hline 17 & & & & & 2.576 & 1.944 & 3.033 & 2.072 & 1.081 & 4.271 & 8.091 & 9.671 & 0.999 \\
\hline 18 & & & & & & 5.685 & 1.123 & 8.323 & 3.882 & 1.631 & 5.261 & 8.431 & 9.601 \\
\hline 19 & & & & & & $1.54 \underline{5}$ & 3.004 & 3.433 & 1.632 & 5.932 & 2.081 & 5.881 & 8.711 \\
\hline 20 & & & & & & 3.806 & 1.274 & 1.373 & 7.223 & $2.57 \underline{2}$ & 7.882 & 2.371 & 6.071 \\
\hline 21 & & & & & & & 3.825 & 5.144 & 3.183 & 1.202 & 3.522 & 9.452 & 2.481 \\
\hline 22 & & & & & & & 1.065 & 1.804 & 1.343 & $5.67 \underline{3}$ & 1.712 & 4.382 & 1.051 \\
\hline 23 & & & & & & & 2.726 & 5.825 & 5.294 & 2.613 & $8.51 \underline{3}$ & 2.192 & 5.052 \\
\hline 24 & & & & & & & & 1.735 & 1.944 & $1.14 \underline{3}$ & 4.173 & 1.132 & 2.602 \\
\hline 25 & & & & & & & & 4.766 & 6.595 & 4.634 & $1.97 \underline{3}$ & 5.843 & 1.392 \\
\hline 26 & & & & & & & & 1.236 & $2.06 \underline{\underline{z}}$ & $1.75 \underline{4}$ & 8.734 & 2.933 & 7.443 \\
\hline 27 & & & & & & & & & & 6.115 & 3.614 & 1.403 & 3.913 \\
\hline 28 & & & & & & & & & & 1.975 & 1.394 & 6.234 & 1.973 \\
\hline 29 & & & & & & & & & & & 4.905 & 2.584 & 9.394 \\
\hline 30 & & & & & & & & & & & 1.605 & 9.925 & 4.164 \\
\hline 31 & & & & & & & & & & & $4.86 \underline{6}$ & $3.51 \underline{5}$ & 1.714 \\
\hline 32 & & & & & & & & & & & & $1.15 \underline{5}$ & 6.525 \\
\hline 33 & & & & & & & & & & & & $3.51 \underline{6}$ & 2.305 \\
\hline
\end{tabular}




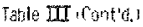

$37^{10^{85}}$ TARget nuclcus)

4I No 93 i TARGet NUCLEUS?

\begin{tabular}{|c|c|c|c|c|c|c|c|c|c|c|c|c|c|c|}
\hline 2 & 6 & 8 & 10 & 12 & 14 & $1 \mathrm{~b}$ & 18 & 22 & $2 \hbar$ & 30 & 34 & 38 & 42 & 46 \\
\hline 0 & 8.036 & 3.613 & 1.501 & 7.081 & 9.431 & 9.871 & $9.96 ?$ & 9.991 & 10,001 & 10.001 & 10.001 & 10.001 & 10.001 & w0.001 \\
\hline 1 & 6.596 & 2.973 & 1.291 & 6.771 & 9.351 & 0.851 & 9.861 & 10.601 & 10,001 & 10,001 & 10,001 & 10.001 & 10.001 & $10.00]$ \\
\hline 2 & 4,446 & 2,023 & 0.412 & 6.071 & 9.181 & $9,82 !$ & 0.051 & 9.991 & 10,001 & 18.001 & 10.001 & 10,001 & 10.001 & 10.001 \\
\hline 3 & 2.586 & 1.153 & 5.822 & 4.961 & $8.82 ?$ & 9.741 & 9.931 & 9.901 & 10001 & 10.001 & 10.001 & 10.001 & 10,001 & 10.001 \\
\hline 4 & 1.276 & 5.594 & 3.012 & 3.483 & 8.161 & $9.59]$ & 9.891 & $9.99 \mathrm{~g}$ & 10.001 & $10,00 !$ & 10,001 & 10.003 & 10.04] & 10.001 \\
\hline 5 & 5.247 & 2.344 & 1.342 & 2001 & 6.911 & 0.211 & 9.821 & 0.981 & 10601 & 10,001 & 10,001 & 10001 & 10.001 & $10.00 ?$ \\
\hline 6 & & 9.269 & 5.193 & 9.239 & 5.001 & 8.591 & 9.641 & 9.971 & 10,001 & 10,601 & $10.60 !$ & lu.eul & 10.001 & 10.00? \\
\hline 7 & & 3.375 & 1.853 & 3.532 & 2.771 & 7.211 & 0.271 & 9.931 & 9.991 & 10.001 & 10.001 & 10.001 & 10.001 & ln.001 \\
\hline 8 & & 1,175 & 6.314 & $1,21 ?$ & 1.161 & 4.851 & 8.301 & 9.871 & 0.981 & 9.991 & 10.001 & 10.601 & 10,001 & 10.001 \\
\hline 9 & & 3.896 & 2.134 & 3.903 & 3.952 & 2.391 & 6.351 & 9.641 & 0.071 & $10 \mathrm{ml}$ & 10.001 & 10.001 & 10.601 & 10,001 \\
\hline 10 & & 1.236 & 7.255 & 1.273 & 1.212 & 8.622 & 3.561 & 9.111 & 9.891 & 0,981 & 10.001 & 10001 & 10.00! & 10.001 \\
\hline$n$ & & & 2.445 & 4.160 & 3.593 & 2.712 & 1.391 & 7.641 & 0.761 & 8.961 & 0,9 & 10,001 & 10.001 & 100.093 \\
\hline 12 & & & & 1.374 & 1.063 & 8.203 & 4.522 & $4.79 ?$ & 9.211 & 9.921 & 9.981 & 0.991 & 10,001 & 10.001 \\
\hline 13 & & & & 4.415 & 3.084 & 2.543 & 1.402 & 2.081 & 7.661 & 9.651 & 9.971 & 10,001 & 9.991 & 9.991 \\
\hline 14 & & & & 1.365 & 8.615 & 3.894 & 4.533 & 0.912 & 4.791 & 8,941 & $9.82 \mathrm{I}$ & 0.981 & 10.001 & loone \\
\hline 15 & & & & 3.890 & 2.265 & 2.374 & 1.493 & 2.312 & 1.931 & 7.041 & 9.511 & 9.391 & 9.971 & 10,001 \\
\hline 16 & & & & 1.966 & 5.546 & 6.755 & 4,784 & 8.253 & 6.882 & 3.501 & 8.301 & 2.751 & 9.931 & 0.071 \\
\hline 17 & & & & & & 1.705 & 1.454 & 3.013 & 2.482 & 1.211 & 5.241 & 8.391 & $9.86 !$ & 9,071 \\
\hline 18 & & & & & & 4.446 & 4,135 & 1.0083 & 9.763 & 3.542 & 2.341 & 6.401 & 0,141 & 0,001 \\
\hline 19 & & & & & & & & 3.654 & 3.913 & 1.032 & 8,802 & 3.241 & $7.18 !$ & 0.251 \\
\hline 20 & & & & & & & & 1.154 & 1.523 & 3033 & 3.662 & 1.251 & 3.341 & 7.691 \\
\hline 21 & & & & & & & & 3.335 & 5.574 & 8.534 & 1.672 & 5.342 & 1.381 & 4,361 \\
\hline 22 & & & & & & & & & 1.904 & 2.254 & 7.753 & 2.532 & 6.442 & 1.821 \\
\hline 23 & & & & & & & & & 5,65 & 5.515 & 3.443 & 1.242 & 3.382 & 8.282 \\
\hline 24 & & & & & & & & & & 1.255 & 1.493 & 5.973 & 1.722 & 4.15? \\
\hline 25 & & & & & & & & & & 2.596 & $5.90 A$ & 2773 & 8.793 & 2.182 \\
\hline 26 & & & & & & & & & & $4,65 ?$ & 2.214 & 1.213 & 4353 & 1.162 \\
\hline 27 & & & & & & & & & & & & 4.024 & 2.043 & 0.043 \\
\hline 28 & & & & & & & & & & & & 1.859 & 8.984 & 3.023 \\
\hline 29 & & & & & & & & & & & & 6.465 & 3.674 & 1. 423 \\
\hline 30 & & & & & & & & & & & & 2.093 & 1.394 & 6.214 \\
\hline 31 & & & & & & & & & & & & 0.236 & 0,875 & 2.534 \\
\hline 32 & & & & & & & & & & & & & 1.585 & 0.515 \\
\hline 33 & & & & & & & & & & & & & 4.806 & 3.325 \\
\hline
\end{tabular}

\begin{tabular}{|c|c|c|c|c|c|c|c|c|c|c|c|c|c|}
\hline$\ell$ & 8 & 10 & 12 & 14 & 16 & 18 & 22 & 26 & 30 & 34 & 38 & 42 & 46 \\
\hline 0 & 4.724 & 3.532 & 4.201 & 8,641 & $9.71 !$ & 9.931 & 9.991 & 10.001 & 10,001 & 10.001 & 10.001 & 10,001 & 10.001 \\
\hline 1 & 3.944 & 2.90 & $3.84 !$ & 8,471 & 9.671 & 9.921 & 0.991 & 10.001 & 10,001 & 10.001 & 10.001 & 10.001 & 10.001 \\
\hline 2 & 2.764 & 2.152 & 3.141 & 8.101 & 9.601 & 9.001 & 9.991 & 10.001 & $10.00 \underline{\underline{n}}$ & 10.000 & 10.001 & 30,001 & 10.001 \\
\hline 3 & 1.654 & 1.312 & 2.251 & 7.401] & 9.431 & 0.861 & 9.991 & 10.001 & 10.001 & 10,001 & 10,001 & 10.001 & 10.001 \\
\hline 4 & 8.595 & 6.853 & 1.361 & 0.261 & 9.121 & 9.781 & 9.981 & 10.001 & 10.001 & 10.001 & 10.001 & 10.001 & 10.001 \\
\hline 5 & 3.975 & 3.153 & 6.932 & 4.591 & 8.501 & $9,64]$ & 9.971 & $9.99 !$ & 30.001 & 10.001 & 10.001 & 10,000 & 10.001 \\
\hline 6 & 1.685 & 1.303 & $2.48 ?$ & 2.751 & 7.321. & 0.311 & 9.551 & 9.991 & 10.001 & 10.000 & 10.001 & 10.001 & 10.601 \\
\hline$i$ & 6.626 & 5.044 & 1.112 & 1.291 & 5.371 & 8.631 & $9.8 \% 1$ & 9.991 & 10,001 & 10.001 & 30.001 & 10.000 & 10.001 \\
\hline 8 & 2.446 & 1.881 & $3.75 \underline{3}$ & 4.092 & 3,021 & 7.151 & 9781 & 9.971 & 0.991 & 10.001 & 10.001 & 10.001 & 10.001 \\
\hline y & $8.06 ?$ & 0885 & 1.173 & 1.682 & 1.291 & 4.761 & 9.441 & 9.951 & 9.991 & 10.001 & $10.00 !$ & 10.001 & 10.001 \\
\hline 16 & & 2,465 & 3.564 & 5.293 & 4.472 & 2.301 & 8.621 & 9.851 & 9.981 & 10.001 & 10,001 & 10,001 & 10,001 \\
\hline 11 & & 8.506 & 0.035 & 1.623 & 1.432 & 8.392 & 6.721 & 9.641 & 0.951 & 9.991 & 10.009 & 10.000 & $10.00 !$ \\
\hline 12 & & & 2705 & 4.854 & 4.493 & 2.752 & 3.801 & 8.941 & 9.891 & 9.981 & 0.091 & 10,001 & 10.001 \\
\hline 13 & & & 0.860 & 1.004 & 1.403 & 8.793 & 1.541 & $7.10 \mathrm{I}$ & 9.611 & 9.961 & 9.991 & 9.991 & 10.001 \\
\hline 14 & & & 1.036 & 3.855 & 4.314 & 2.873 & 5.212 & 4.161 & 8761 & 9.811 & 9.981 & 10.001 & 10.001 \\
\hline 15 & & & & 9.946 & 1,274 & 9.364 & 1.782 & 1.601 & 6.711 & 9.421 & 9.891 & 9.981 & 10.001 \\
\hline 16 & & & & 2.356 & 3.525 & 2.044 & 6.383 & 5.912 & 3.441 & 8.221 & 9.711 & 9.921 & 9.981 \\
\hline 17 & & & & & 9.036 & 8.705 & 2303 & 2,202 & L.351 & 5.231 & 8.051 & 9.851 & 9.351 \\
\hline 18 & & & & & $2.19 \%$ & 2.405 & 8.024 & 8.583 & 5.0992 & 2.361 & 6.561 & 9.281 & 9.911 \\
\hline 19 & & & & & & & 2.644 & 3.383 & $207 ?$ & 9.172 & $3.44]$ & 7.421 & 9.421 \\
\hline 20 & & & & & & & 8125 & 1.283 & 8.843 & 3.812 & 1.381 & 4.361 & 7.961 \\
\hline 21 & & & & & & & 2.325 & 4.564 & 3.733 & 1.722 & 5.842 & 1.83! & 5.001 \\
\hline 22 & & & & & & & 6.116 & 1.524 & 1.503 & 7.823 & 2.732 & 7.892 & 2.181 \\
\hline 23 & & & & & & & 1.466 & 4.665 & 5.649 & 3.463 & 1.322 & 3.802 & 9.712 \\
\hline 24 & & & & & & & & & 1.984 & 1.453 & 6.283 & 1.922 & 4.802 \\
\hline 25 & & & & & & & & & 6.415 & 5.644 & 2.863 & 9.663 & 2.512 \\
\hline 26 & & & & & & & & & 1.935 & 2.054 & 1.223 & 4.713 & 1.322 \\
\hline 27 & & & & & & & & & & $6.87 \underline{2}$ & $4.87 \pi$ & 2.173 & 6.793 \\
\hline 28 & & & & & & & & & & 2.135 & 1.804 & 9.374 & 3.343 \\
\hline 29 & & & & & & & & & & & 6.165 & 3.764 & 1.543 \\
\hline 30 & & & & & & & & & & & 1.965 & 1.404 & 6.654 \\
\hline 31 & & & & & & & & & & & 5.756 & 4.835 & 2.668 \\
\hline 32 & & & & & & & & & & & & 1.555 & 9.865 \\
\hline 33 & & & & & & & & & & & & 4.6616 & 3.395 \\
\hline 34 & & & & & & & & & & & & & 1.095 \\
\hline 35 & & & & & & & & & & & & & 3.235 \\
\hline
\end{tabular}


Iafiet III Cont'

\begin{tabular}{|c|c|c|c|c|c|c|c|c|c|c|c|c|c|c|}
\hline$\ell$ & 8 & 10 & 12 & 14 & 16 & 18 & 20 & 22 & 26 & 30 & 39 & 38 & 42 & 46 \\
\hline 0 & 6.775 & 7.703 & 1.761 & 7.081 & 9.391 & 0.851 & 9.961 & 9.991 & 10.001 & 10.001 & 10.001 & 10001 & 10.001 & 10.001 \\
\hline 1 & 5.785 & 6.553 & 1.55d & $6.79 \underline{1}$ & 9.311 & 9.841 & 9.961 & 9.991 & $10.00 \mathrm{I}$ & 10.001 & 10.001. & 10.001 & 10.001 & 10.001 \\
\hline 2 & 4.223 & 4.763 & 1.191 & 6.201 & 9.151 & 9.801 & 9.941 & 0,081 & 10.003 & 10.001 & 10,001 & 10.001 & 10.001 & 10,001 \\
\hline 3 & 2.605 & 2.983 & 7.942 & 5.221 & 8.831 & 9.731 & 9.931 & 9.981 & 10.001 & 10.001 & 10,001 & 10.001 & 10.001 & 10.001 \\
\hline 4 & 1.495 & 1.633 & 4532 & 3.911 & $8.26 !$ & $9.5 \% 1$ & 9.891 & 9.971 & 10000 & 10.001 & 10.001 & 10.001 & 10.001 & 10.001 \\
\hline 5 & 7.560 & 7.944 & 2.232 & 2.481 & 7.221 & 9.331 & $9.83 \mathrm{~B}$ & 9.951 & 9.991 & 10,001 & 106001 & 10.001 & 10.001 & 10.001 \\
\hline 6 & 3.456 & 3.544 & 9.543 & 1.301 & 5.581 & 8.171 & 9.681 & 9.911 & 9.991 & 10.001 & 10,001 & 10.001 & 100001 & 10.001 \\
\hline 7 & 1.486 & 1.504 & 3.643 & 5.662 & 3.531 & 7.691 & 9.381 & 9.821 & 9.981 & 10.001 & 10.001 & 10.001 & 10001 & 10001 \\
\hline 8 & 6.117 & 6005 & 1.263 & $2.1,2 ?$ & $1.74 !$ & 5.761 & 8.701 & 9.643 & 9.461 & 9.991 & 10.001 & 10.001 & 10.001 & 10.001 \\
\hline 9 & & 2395 & 4.084 & 1.393 & $6.92 ?$ & $3.36 !$ & $7.22 !$ & 9.161 & 9.921 & 9.991 & 10.001 & 10.001 & 10.001 & $10,00 !$ \\
\hline 10 & & 9.206 & 1.244 & 2,393 & 2.402 & $1.47 !$ & 4.831 & 8.041 & 9.801 & 9.983 & 10.001 & 10.001 & 10.001 & 10.001 \\
\hline 11 & & 3.446 & 3.545 & 7.464 & 7.863 & 5.272 & 2.331 & 5.841 & 9.501 & 0.941 & 9.991 & 10001 & 10,01 & 10.001 \\
\hline 12 & & & 9.5016 & 2.244 & 2.513 & $1.75 ?$ & $8.79 ?$ & 3061 & 8.681 & 9.851 & 9.971 & 9991 & 10.001 & 10.001 \\
\hline 13 & & & 2.406 & 6.395 & 7.864 & $5.73 ?$ & 2.982 & $1.2 \mathrm{ll}$ & 6.661 & $9.50 !$ & 9.051 & 0.991 & 10.001 & 10.001 \\
\hline 14 & & & & & 2394 & 1.873 & 9.993 & 4.162 & 3.761 & 8.631 & 9.821 & 9.981 & 10.001 & 10.001 \\
\hline 15 & & & & & 6.885 & 6.004 & 3.403 & 1.442 & 1.521 & 6.541 & 9.391 & a yol & 9.991 & 10.001 \\
\hline 16 & & & & & 1.865 & 1.844 & 1.153 & 5.113 & 5.472 & 3.441 & $8.24 !$ & 9,801 & 9.931 & $9.99 !$ \\
\hline 17 & & & & & & 5.305 & 3.724 & 1.813 & 2.042 & $13 / 1$ & 5.461 & 9.071 & 9.841 & 9.051 \\
\hline 18 & & & & & & 1.425 & 1.134 & 6.184 & 7.873 & $5.2 \mathrm{~d} 2$ & $2.55 !$ & 6.951 & 9.441 & 9.911 \\
\hline 19 & & & & & & & 3.235 & 1.974 & 3.023 & 2.122? & 1,061 & $3.45 !$ & $7.80 !$ & $9.61 !$ \\
\hline 20 & & & & & & & 8.516 & 5.985 & 1.123 & 8.843 & 4.202 & 1.631 & 4.991 & 8.391 \\
\hline 21 & & & & & & & 2.116 & 1.645 & 3.884 & 3.643 & 1.862 & 6.832 & 2.261 & 5.851 \\
\hline 22 & & & & & & & & 4.236 & 1.264 & 1.433 & 8.303 & 3.132 & 9.652 & 2.811 \\
\hline 23 & & & & & & & & 1.026 & 3.775 & 5.254 & 3.593 & 1.442 & 4.552 & $1.23 !$ \\
\hline 24 & & & & & & & & & & 1.804 & 1.473 & 0. 943 & 2.262 & 5.942 \\
\hline 25 & & & & & & & & & & 5.705 & 5604 & 3.103 & 1.122 & 3.052 \\
\hline 26 & & & & & & & & & & $1.67 \underline{\text { s. }}$ & 1.994 & 1303 & 5363 & 1.592 \\
\hline 27 & & & & & & & & & & & 6.535 & 5.064 & 2.433 & 8053 \\
\hline 28 & & & & & & & & & & & 1.995 & $1.89 \mathrm{k}$ & 1.033 & $389 \underline{3}$ \\
\hline 29 & & & & & & & & & & & & 6.175 & 4,064 & 1.773 \\
\hline 30 & & & & & & & & & & & & 1.935 & 1.484 & 7.494 \\
\hline 31 & & & & & & & & & & & & 5.586 & 5.035 & 2.954 \\
\hline 32 & & & & & & & & & & & & & 1.585 & 1.084 \\
\hline 33 & & & & & & & & & & & & & 4.656 & 3.655 \\
\hline 34 & & & & & & & & & & & & & & 1.155 \\
\hline 35 & & & & & & & & & & & & & & 3.396 \\
\hline
\end{tabular}

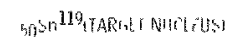

\begin{tabular}{|c|c|c|c|c|c|c|c|c|c|c|c|c|c|}
\hline 2 & 10 & 12 & 1.4 & 16 & 18 & 20 & 22 & 26 & 30 & 34 & 38 & 42 & ab \\
\hline 0 & 1283 & 4.812 & 4.271 & $8.54 \mathbf{I}$ & 0.671 & 9.911 & 9.971 & 10,01 & 100001 & 100001 & 10.60? & 10,001 & $2 n, 00$ \\
\hline 1 & 1.103 & 4.202 & 3961 & 8.391 & 9,041 & 9.901 & 9.971 & 10.001 & 10,001 & $10.00 \mathrm{I}$ & 10.001 & 10.901 & 10.001 \\
\hline 2 & 8.284 & 3.202 & 3,361 & $8.06 ?$ & $0.56]$ & 4.891 & 9.971 & 10001 & $10.00\}$ & $10.00 \mathrm{~g}$ & 10.001 & 10.901 & 10.001 \\
\hline 3 & $5.45 \%$ & 2142 & 2.561 & 7.471 & 9.421 & 9.851 & 0.061 & $9.99 !$ & 10.901 & 10.001 & 10.001 & 10.000 & 10.001 \\
\hline 4 & 3.204 & 1,262 & 1.701 & 6.501 & $9.1 / 1$ & 0.781 & 9.941 & 9.991 & 10.001 & 10.009 & 10.001 & 10001 & 10.001 \\
\hline 5 & 1.704 & 8583 & 9.752 & 5.111 & 8.041 & 9. 601 & 9.001 & 9.991 & 10.601 & 10.001 & 10.001 & 10,001 & 10,001 \\
\hline 6 & 8.345 & 3.143 & 4,822 & 3.421 & 7.721 & 9.411 & $9.84 \mathrm{l}$ & $9.9 \% 1$ & 10.001 & 10.001 & 10.001. & 10.001 & 10.001 \\
\hline 7 & 3.845 & 1.403 & 2082 & 1.591 & 6.181 & 8.901 & 9.701 & 9.971 & 10,001 & 10,001 & 10.001 & $10.09 \mathrm{~L}$ & 10.001 \\
\hline 8 & 1.685 & 5.994 & 8.093 & 8.612 & 4.091 & 7.671 & 9.401 & 9.941 & 9.991 & 10.601 & 10.001 & 10.000 & 10.001 \\
\hline$a$ & 7.146 & $2,50 \mathrm{ph}$ & 2.893 & 3.392 & 2.131 & 0.031 & 8.721 & $9.88 \mathrm{~g}$ & 0.981 & 10,001 & 10.001 & $10.000 ?$ & 10.001 \\
\hline 10 & 2856 & 1.033 & 9.649 & 1.212 & 8.912 & 36.11 & 7.281 & $9.73 !$ & 9.971 & $9.99 !$ & 10.001 & 10001 & 10.001 \\
\hline 11 & 1.076 & 4.155 & 3.054 & 4.103 & 3.52 & 1.671 & 4.941 & $9.34 \mathrm{l}$ & 9.931 & 9.991 & 10.091 & 10.91 & 10,001 \\
\hline 12 & & 1.665 & 9.115 & 1.33 & 1.112 & 6.342 & $2.49 !$ & 8.391 & 9.811 & 9.971 & 10,001 & 10.001 & 10.0in \\
\hline 13 & & 0.486 & 2575 & 4.154 & 3.713 & 2.222 & 9.882 & $6.34 \mathrm{k}$ & 9.501 & $9.93 !$ & 9.991 & 160001 & 10.001 \\
\hline 14 & & 2.426 & 6.776 & 1234 & 1.203 & 7.573 & 3.532 & 3.581 & 8.011 & 9.821 & 9971 & 9.991 & 10.001 \\
\hline 15 & & & 1650 & 3.465 & 3.778 & 2.553 & 1.242 & 1.521 & 6.601 & 9., $m 41$ & 9.921 & $9.99 \%$ & 10.001 \\
\hline 16 & & & 3.587 & 9.086 & 1.124 & 8394 & 4.343 & 5.672 & $3.74 !$ & 8401 & 9.741 & 0.961 & 10.001 \\
\hline 17 & & & 9.708 & 2.226 & 3.135 & 2,634 & 1.493 & 2.122 & 1.591 & 6.071 & 9.221 & $9.86 !$ & 9.971 \\
\hline 18 & & & & & 8.136 & 7.775 & 4.9004 & 7.993 & 0.242 & 3.141 & 7.681 & 0.591 & 9.921 \\
\hline 19 & & & & & 1.966 & 2.145 & 1.524 & 2.973 & 2.492 & 1.331 & 4,801 & 8.581 & 9.761 \\
\hline 20 & & & & & & 5.476 & 4.405 & 1.063 & $1.01 ?$ & 5.512 & 2.271 & 6.161 & 9.051 \\
\hline 21. & & & & & & 1.286 & 1.185 & 3.564 & 0.023 & 2.372 & 9.712 & 3.271 & 7.121 \\
\hline 22 & & & & & & & 2.376 & 1.124 & 1.523 & 1.022 & 4.332 & 1,461 & 4.181 \\
\hline 23 & & & & & & & & 3.265 & 5.439 & 4293 & 1.992 & 6.682 & 1.041 \\
\hline 24 & & & & & & & & 8.826 & 1.804 & 1.703 & 9.033 & 3.222 & 9.132 \\
\hline 25 & & & & & & & & 2226 & 5.515 & 6.304 & 3.913 & 1.562 & 4.562 \\
\hline 26 & & & & & & & & & 1.605 & 2.184 & 1.603 & 7.263 & 2.322 \\
\hline 27 & & & & & & & & & & 6.995 & 6.074 & 3.203 & $1.15 ?$ \\
\hline 28 & & & & & & & & & & 2.095 & 2.154 & 1.333 & 5.433 \\
\hline 29 & & & & & & & & & & & 7.075 & 5.104 & 2.413 \\
\hline 30 & & & & & & & & & & & 2.165 & 1.834 & 9.994 \\
\hline 31 & & & & & & & & & & & 0.186 & 6.085 & 3.864 \\
\hline 32 & & & & & & & & & & & & 1.885 & 1.384 \\
\hline 33 & & & & & & & & & & & & 5.426 & 4.615 \\
\hline 34 & & & & & & & & & & & & & 1.435 \\
\hline 35 & & & & & & & & & & & & & 4.186 \\
\hline 36 & & & & & & & & & & & & & 1.136 \\
\hline
\end{tabular}


Table III (Cont'd )

$60 \mathrm{Nd}^{144}$ (TARGET NUCLEUS)

\begin{tabular}{|c|c|c|c|c|c|c|c|c|c|c|c|c|c|c|c|}
\hline$\ell$ & 8 & 10 & 12 & 14 & 16 & 18 & 20 & 22 & 24 & 26 & 30 & 34 & 38 & 42 & 46 \\
\hline 0 & 4478 & 2605 & 1843 & 4502 & 3711 & 8111 & 9561 & 9881 & 9961 & 9991 & 10001 & 10001 & 10001 & 10001 & 10001 \\
\hline 1 & 5968 & 2315 & 1633 & 4012 & 3461 & 7951 & 9521 & 9871 & $996 \underline{1}$ & 9991 & 10001 & 10007 & 10001 & 10001 & 10001 \\
\hline 2 & 2988 & $185 \underline{5}$ & $129 \underline{3}$ & $318 \underline{2}$ & 2971 & 7601 & 9421 & 9851 & 9951 & 9981 & 10001 & 10001 & 10001 & 10001 & 10001 \\
\hline 3 & 2988 & I 325 & $909 \underline{4}$ & $225 \underline{2}$ & 2321 & 7011 & 9261 & 9811 & 9941 & 9981 & 10001 & 10001 & 10001 & 10001 & 10001 \\
\hline 4 & & 8606 & 5824 & 1432 & 1631 & 6091 & 8961 & 9731 & 9921 & 9971 & 10001 & 10001 & 10001 & 10001 & 10001 \\
\hline 5 & & 5166 & 3424 & 8153 & 1011 & $484 \underline{1}$ & $844 \underline{I}$ & 9591 & 9881 & 9961 & 10001 & 10001 & 100001 & 10001 & 10001 \\
\hline 6 & & 2856 & 1874 & 4263 & 5622 & 3391 & $75 \underline{5}$ & $934]$ & $981 \underline{1}$ & 9941 & 9991 & 10001 & 10001 & $10 \mathrm{col}$ & 10001 \\
\hline 7 & & 1436 & 9665 & 2073 & 2822 & 2031 & 6151 & 8851 & 9681 & 9901 & 9991 & $1000 \mathrm{I}$ & 10001 & 10001 & 10001 \\
\hline 8 & & 7307 & 4735 & 9424 & 1312 & 1051 & 4311 & 7921 & $940 \mathrm{I}$ & 9821 & 9981 & 10000 & 10001 & 10001 & 10001 \\
\hline 9 & & & 2225 & $407 \underline{4}$ & 5753 & 4832 & 2491 & 6341 & 8811 & 9641 & 9961 & 9991 & 10001 & 10001 & 10001 \\
\hline 10 & & & 1005 & 1674 & 2443 & 2082 & $121 \underline{1}$ & 4211 & 7631 & 9251 & 9921 & 9991 & 10001 & 10001 & 10001 \\
\hline 11 & & & 4376 & 6515 & 1013 & 8663 & 5202 & 2231 & 5661 & $837 \underline{1}$ & 9821 & 9981 & 10001 & 10001 & 10001 \\
\hline 12 & & & 1826 & 2395 & 4054 & 3583 & 2142 & 9952 & 3321 & 6661 & 9591 & 9951 & 9991 & 10001 & 10001 \\
\hline 13 & & & & 8326 & 1564 & $146 \underline{3}$ & 8823 & $410 \underline{2}$ & 1551 & 4231 & 9001 & 9871 & 9981 & $10 \mathrm{col}$ & 10001 \\
\hline 14 & & & & 2686 & 5735 & 5874 & $366 \underline{3}$ & 1692 & 6492 & 2091 & 7641 & 9681 & 9951 & 9991 & 10001 \\
\hline 15 & & & & & 1995 & 2274 & $152 \underline{3}$ & 7083 & 2672 & $\delta 872$ & 5271 & 9141 & 9881 & 9981 & 10001 \\
\hline 16 & & & & & 6535 & 8405 & 6164 & 3023 & 1142 & 3682 & 2771 & 7811 & 9661 & 9951 & 9991 \\
\hline 17 & & & & & 1906 & 2935 & 2414 & 1273 & 4963 & 1582 & 1211 & 5391 & 9021 & $984 \underline{1}$ & 9973 \\
\hline 18 & & & & & & 9636 & 8935 & 5234 & 2173 & $707 \underline{3}$ & 5142 & 2801 & 7451 & $953]$ & 9921 \\
\hline 19 & & & & & & 2996 & 3135 & 2054 & 9284 & 3193 & 2302 & $124]$ & 4781 & 8621 & 9751 \\
\hline 20 & & & & & & & 1045 & 7615 & 3824 & I 423 & 1082 & 5502 & $23 / 1$ & 6501 & 9211 \\
\hline 21 & & & & & & & 3196 & 2665 & 1494 & 6094 & 5133 & 2582 & 1071 & 3721 & 767 \\
\hline 22 & & & & & & & & 8726 & 5495 & 2494 & 2433 & 1272 & 5002 & 1781 & 5011 \\
\hline 23 & & & & & & & & $26 d \underline{6}$ & 1005 & 9605 & 1123 & o 333 & $247 ?$ & 8332 & 2571 \\
\hline 24 & & & & & & & & & 6146 & 3485 & 4914 & 3153 & 1272 & 4142 & I 231 \\
\hline 25 & & & & & & & & & 1856 & 1195 & 2054 & 1533 & 6633 & 2162 & 6182 \\
\hline 26 & & & & & & & & & & 3780 & 8045 & 7114 & 3433 & 1162 & 3262 \\
\hline 27 & & & & & & & & & & & 2965 & 3144 & 1733 & 6233 & 1772 \\
\hline 28 & & & & & & & & & & & 1025 & 1314 & 8434 & 3333 & 9813 \\
\hline 29 & & & & & & & & & & & & & 3904 & 1743 & 5453 \\
\hline 30 & & & & & & & & & & & & & 1704 & 8744 & 2993 \\
\hline 31 & & & & & & & & & & & & & 6985 & 4184 & 1603 \\
\hline 32 & & & & & & & & & & & & & & 1884 & 8244 \\
\hline 33 & & & & & & & & & & & & & & 7985 & 4034 \\
\hline 34 & & & & & & & & & & & & & & & 1864 \\
\hline 35 & & & & & & & & & & & & & & & 8055 \\
\hline 36 & & & & & & & & & & & & & & & 3275 \\
\hline
\end{tabular}


Idale III cont'd I

$70 \times b^{173}$ (TARGET NUCLUUS

$78^{197^{19}}$ I IARGL I MUCLUUS:

\begin{tabular}{|c|c|c|c|c|c|c|c|c|c|c|c|c|c|c|c|c|c|c|c|c|c|c|c|c|c|c|}
\hline l. & 12 & 14 & 16 & 18 & 20 & 22 & 24 & 26 & 30 & 34 & 38 & 42 & 46 & $\ell$ & 14 & 16 & 18 & 20 & 22 & 24 & 20 & 30 & 34 & 38 & 42 & 46 \\
\hline 0 & 8.075 & 3063 & 5.212 & 3.651 & 7.921 & 9.481 & 9.861 & 9.961 & 9.991 & 10.001 & 10.001 & 10.001 & 10.001 & 0 & 3,344 & 1.593 & 9.032 & 4.571 & 8.311 & 9.561 & 9.871 & 9.991 & 10.003 & 10.001 & 10.001 & 10.001 \\
\hline 1 & 7.325 & 2.763 & 4.722 & 3.421 & $7.7 n 1$ & 9441 & 9.851 & 0.951 & 9.991 & 10.001 & 10.001 & 10,002 & 10001 & 1 & 3069 & 6.933 & 8.292 & 4.361 & 8.191 & 9.531 & 9.871 & 9.981 & 10.001 & 10.003 & 10.001 & 10,001 \\
\hline 2 & 6.045 & 2.263 & 3.872 & 3001 & 7.451 & 9.351 & 9.821 & 9.951 & 9.991 & 10.001 & 10.001 & 10.001 & 10.001 & 2 & 2.584 & 5.783 & 6.982 & 3.931 & 7.941 & 0.451 & 9.851 & 9.981 & $10 . \mathrm{mg}$ & 10.001 & 10.001 & 10.001 \\
\hline 3 & 4.545 & 1.683 & 2.872 & 2.431 & 6.001 & 9.181 & 0.781 & 9.931 & 9.991 & 10.001 & 10.001 & 10.001 & 10000 & 3 & 2.094 & 4433 & 5.392 & 3331 & 7.511 & 0.331 & 0,811 & 9.981 & 10.001 & 10.001 & 10.001 & 10,001 \\
\hline 4 & 3145 & 1153 & 1.942 & 1.791 & 6.091 & 8.901 & 9.703 & 9.911 & 9.991 & 10.001 & 10.001 & 10.001 & 10,001 & 4 & 1.438 & 3.133 & 3.812 & 2.611 & 6861 & 0.111 & 9.751 & 9.971 & 10.001 & 10.001 & 10,001 & 10.001 \\
\hline 5 & 2.025 & 7.264 & 1.202 & 1.191 & 4.991 & 8.421 & 9.571 & $9.87 \underline{1}$ & 9.991 & 10.001 & 10.001 & 10.001 & 10.001 & 5 & 9.575 & 20063 & 2.472 & I. 861 & 5.931 & 8.761 & 9.651 & 0.961 & 9.991 & 10.001 & 10.001 & 10.001 \\
\hline 6 & 1.205 & 4.3724 & 0.793 & 7.202 & 3.691 & $7.64 \mathrm{I}$ & 9.341 & $9.81 \mathrm{I}$ & $9.81 \mathrm{I}$ & $10.00 \mathrm{I}$ & 10.001 & 10.001 & I0.001 & 0 & 0.015 & 1.283 & 1.482 & 1.211 & $4,3 !$ & $8,18 \underline{1}$ & 9.481 & $9,95 !$ & 9.991 & 10.001 & 10.001 & 10,001 \\
\hline 7 & 6.736 & 2.414 & 3.583 & $3 \% 62$ & 2.401 & 6.441 & 8.911 & 9.691 & 9.971 & 10.001 & 10.001 & 10.001 & 10.001 & 7 & 3.555 & 3,4094 & 8.283 & 1.182 & 3341 & 7.261 & $4.17 \underline{1}$ & 9.921 & 9.991 & 10.001 & 10.001 & 100001 \\
\hline 8 & 3.536 & 1.294 & 1.773 & 2.022 & 1.371 & 4.831 & 8.1151 & 9.451 & 9.941 & 9.991 & 10.001 & 10.001 & 10,001 & 8 & 2.015 & 4.214 & 4.353 & 3.922 & 2151 & 5.911 & 8.631 & 9.871 & 9981 & 10.001 & 10.001 & 10.001 \\
\hline$q$ & 1.816 & 6.545 & 8.304 & 9.683 & 7.032 & 3.101 & $6.85 \underline{1}$ & 8991 & 9.901 & 9.491 & 10.001 & 10.001 & 10001 & 9 & 1.075 & 2.284 & 2.163 & 2.002 & 1.211 & 4.251 & 7.671 & 9.771 & 9.971 & 10.001 & 10.001 & 10.001 \\
\hline 10 & & 3.265 & 3.694 & 4.453 & 3.332 & $1.69 \mathrm{I}$ & 5.011 & 8,081 & 9.801 & 9.97 ] & 10.001 & 10.001 & .10 .001 & 10 & 5.476 & 1,194 & 1,023 & 9.753 & 6.192 & 2.621 & 6.182 & 9.571 & 9.951 & 9.991 & 10.001 & 10,001 \\
\hline 11 & & 1.545 & 1.564 & 1.983 & 1.512 & 8.182 & 3041 & 6.521 & 9.591 & 9.951 & 9.991 & 10.001 & 10001 & 11 & 2620 & 6.055 & 4.584 & 4.563 & 2.982 & $1 \mathrm{~m}$ & 4.281 & 9151 & 9.001 & 9.991 & 10.001 & 10.001 \\
\hline 12 & & 7.186 & 6.215 & 8.474 & 6.653 & 3692 & $1.55 \underline{1}$ & $4.40 I$ & 9.131 & 9391 & 9.981 & 10.001 & $10.00 ?$ & 12 & 1.246 & 2075 & 1.964 & 2063 & 1.392 & 6842 & $2.49 \mathrm{I}$ & 8.311 & 9.801 & $9.97 \mathrm{I}$ & 10.001 & 10.001 \\
\hline 13 & & 3.126 & 2.335 & 3.494 & 2.883 & 1.622 & 7.092 & 2.41 .1 & 8.121 & 9.761 & 9.971 & 9.991 & 10.001 & 13 & & 1.425 & 7.925 & 9.004 & 6.283 & 3,172 & 1,261 & 6.791 & 9.571 & 9.941 & 9.991 & 10.001 \\
\hline 14 & & 1.330 & $8.2 \% 6$ & 1.374 & 1.223 & 7.073 & 3.122 & 1.141 & 0.281 & $9.44 \underline{1}$ & 9.922 & 9.091 & 10.001 & 14 & & & 3035 & 3.774 & 2789 & 1.442 & 5872 & $4.66 \mathrm{I}$ & 9,041 & $9,87 \underline{1}$ & 9.983 & 100002 \\
\hline 15 & & & 2.736 & 5.125 & 5.004 & 3063 & $1.37 ?$ & 5.022 & 3.911 & 8.67 & 9.811 & 0.971 & 9.991 & 15 & & & 1.095 & 1,504 & 1.203 & 6.463 & 2.672 & 2.621 & 7.021 & 0.701 & 9.951 & 9.991 \\
\hline 16 & & & & 1.805 & 1.964 & 1.303 & 6.033 & 2.222 & 1.971 & 7.061 & 9.521 & 9,931 & 9.991 & 16 & & & 3.716 & 5.685 & $4.97 \underline{\square}$ & 2.863 & 1,212 & 1.281 & 5.981 & 9.281 & 9.891 & 9.981 \\
\hline 17 & & & & 5.956 & 7.295 & 5.324 & 2.643 & 9.933 & 8.032 & 4.641 & 8.771 & 9.811 & 0.971 & 17 & & & 1.166 & 2.035 & 1.974 & 1.233 & 5.483 & 5.912 & 3.651 & 8.311 & 9.731 & 9.961 \\
\hline 18 & & & & 1.876 & 2565 & 2.084 & 1.133 & 4.473 & 4.012 & 2.431 & 7.131 & 9.471 & 9.911 & 18 & & & & 6.826 & 7.385 & 5.064 & 2.433 & 2.742 & 1.861 & 6.451 & 9.311 & 9.891 \\
\hline 19 & & & & & & 7.75 & 4.614 & 1.973 & 1.862 & 1.131 & 4641 & 8.571 & 9.741 & 19 & & & & & 2615 & 1.994 & 1.043 & 1.282 & 8.782 & $4.03 \underline{\underline{l}}$ & 8.281 & 9.691 \\
\hline 20 & & & & & & 2.695 & 1.799 & 8.424 & 8.753 & 5212 & 2.421 & 6.671 & 9.271 & 20 & & & & & 8.706 & 7.385 & 4.264 & 6.103 & 4.172 & 2,091 & $0.31 \mathrm{I}$ & 9.171 \\
\hline 21. & & & & & & $8.87 \underline{6}$ & 6.605 & 3.434 & 4.113 & $2.50 ?$ & $1.14]$ & $4.139 \underline{1}$ & 8.021 & 21 & & & & & 2.706 & 2.595 & 1.664 & 2.153 & 2.022 & 1.011 & 3.851 & 7.911 \\
\hline 22 & & & & & & & 2.285 & 1.324 & 1.883 & 1.232 & 5.472 & 2.081 & 5.711 & 22 & & & & & & 8.540 & 6.085 & 1.213 & 9.913 & 4.952 & $1,99 !$ & 5.681 \\
\hline 23 & & & & & & & 7.446 & 4.806 & 8.244 & 0063 & 2.742 & 1.011 & $3.22 !$ & 23 & & & & & & 2.676 & 2,105 & 5.084 & 4.783 & 2492 & 9.852 & 3.291 \\
\hline 24 & & & & & & & & 1.645 & 3.444 & 2.933 & 1.402 & 5.042 & 1.622 & 24 & & & & & & & 6.836 & 2.024 & 2.243 & 1272 & 5.002 & 1.691 \\
\hline 25 & & & & & & & & 5.226 & 1.354 & 1.363 & 7.233 & $2.69 ?$ & $8.15 ?$ & 25 & & & & & & & 2.096 & 7.575 & 9.994 & 6.413 & 2.612 & 8.612 \\
\hline 26 & & & & & & & & 1.576 & 5.005 & 6.044 & 3.653 & 1.402 & 4292 & 26 & & & & & & & & 2.675 & 4.234 & 3.143 & 1.382 & 4.552 \\
\hline 27 & & & & & & & & & 1.745 & 2.534 & 1.783 & 7.483 & 2.332 & 27 & & & & & & & & & 1.694 & 1.473 & 7.243 & 2.472 \\
\hline 28 & & & & & & & & & 5.666 & 9.985 & 8.264 & 3.913 & 1.232 & 28 & & & & & & & & & 0.365 & 6.574 & 3.683 & 1.352 \\
\hline 29 & & & & & & & & & & & 3.634 & 1.983 & 7.053 & 29 & & & & & & & & & & 2.766 & 1.803 & 7.293 \\
\hline 30 & & & & & & & & & & & 1.504 & 9.514 & 3.793 & 30 & & & & & & & & & & 1.094 & 8.314 & 3.813 \\
\hline 31 & & & & & & & & & & & 5.845 & 433 & 1.903 & 31 & & & & & & & & & & 4.075 & 3.634 & 1,913 \\
\hline 32 & & & & & & & & & & & & 1,864 & 9.704 & 32 & & & & & & & & & & & 1.494 & 9.104 \\
\hline 33 & & & & & & & & & & & & 7.505 & 4544 & 33 & & & & & & & & & & & 5.775 & 4.094 \\
\hline 34 & & & & & & & & & & & & & 2.004 & 34 & & & & & & & & & & & & 1.734 \\
\hline $\begin{array}{l}35 \\
36\end{array}$ & & & & & & & & & & & & & $\begin{array}{l}8.265 \\
3.215\end{array}$ & $\begin{array}{l}35 \\
36\end{array}$ & & & & & & & & & & & & $\begin{array}{l}6.875 \\
2.575\end{array}$ \\
\hline
\end{tabular}


Table III icont't:

$82 \mathrm{P}^{2006}$ i Aarget nUCLEUS:

0007h 232i TARGE NUCLILS

\begin{tabular}{|c|c|c|c|c|c|c|c|c|c|c|c|c|c|c|c|c|c|c|c|c|c|c|c|c|c|}
\hline$\ell$ & 14 & 16 & 18 & 20 & 22 & 24 & 26 & 30 & 34 & 38 & 42 & 46 & $\ell$ & 14 & 16 & 18 & 20 & 22 & 24 & 26 & 30 & 34 & 38 & 42 & 46 \\
\hline 0 & 1.134 & 2883 & 3.872 & 2.781 & 7.121 & 9.221 & 9.781 & 9,981 & 10.001 & 10.001 & 10.001 & 10.001 & 0 & 1.425 & 4.934 & 6973 & 7.872 & 3071 & 7.841 & $9 ., 01$ & Q.941 & 9.991 & 10.001 & 10.0in] & 10.001 \\
\hline 1 & 1.044 & 2.643 & 3.542 & 2.611 & $6.95 \underline{I}$ & 9.161 & $9.77 \underline{1}$ & 9.981 & 10.002 & 10.001 & 10.01 & 10.001 & 1 & 1325 & $44 \leq 4$ & 6.4013 & 7.3. & 3.791 & 7.721 & 9.361 & 9.941 & 9.991 & 10.001 & 10.001 & 10.001 \\
\hline 2 & 8.935 & 2.233 & 2.982 & 2.291 & 6.601 & 9.041 & 9.741 & 9.971 & 10,001 & I0.00I & 10.001 & 10,001 & $?$ & 1.145 & 3.864 & 54013 & 0.282 & 3.441 & 7.461 & 9.271 & 9.931 & $999 \underline{1}$ & 10,001 & 10.001 & 10.001 \\
\hline 3 & 6.915 & 1.743 & 2.292 & 1.871 & 0.051 & $8.83 \underline{1}$ & 9.081 & $9.97 \underline{?}$ & 10.001 & 10.001 & $10.0 \mathrm{~m}$ & 10,001 & 3 & 9.098 & 3104 & 4203 & 5.002 & 2.941 & 7.031 & $9.13 \mathrm{I}$ & 0.921 & 9.991 & 10.001 & 10.001 & 10.001 \\
\hline 4 & 5.075 & 1.263 & 1.622 & 1.411 & 5.261 & 8.491 & 9.581 & 9.961 & $9.99 !$ & 10.001 & 10.001 & 10.m! & 4 & 6800 & 2.324 & 3013 & 3.628 & 2.351 & 6.401 & 8.891 & 9.901 & 9.991 & 10.001 & 10.001 & 10.001 \\
\hline 5 & $3.40 \underline{5}$ & 8.499 & 1.062 & 9.732 & 4.271 & 7.351 & 9.421 & 9.941 & 0.9911 & 11,001 & 10.001 & $10,00 \underline{\underline{I}}$ & 5 & 4746 & 3.634 & 2003 & 25.52 & $1.74 \underline{n}$ & 5541 & 8.521 & $9.86 !$ & 9.981 & 10.0101 & 10,001 & 10.001 \\
\hline 6 & 2.205 & 5.414 & 6.423 & 6.182 & $3.17 \underline{1}$ & 7.131 & 9.141 & 9.921 & 0,901 & 10,001 & 10,001 & 10.001 & 6 & 3060 & 1.098 & 1.233 & 1.602 & 1.191 & 4.881 & $i, 931$ & 9.811 & 9.981 & 10.001 & $10.00 \mathrm{l}$ & 10.001 \\
\hline 7 & 1.335 & 3.274 & 3.633 & 3.632 & 2.121 & 5.961 & $8.67 !$ & $9.87 \underline{1}$ & 9.981 & 10.001 & 10.001 & 10.001 & 7 & 1.886 & 0875 & 7.091 & 9533 & $7.45 ?$ & 3.311 & 7.061 & 9.711 & 9.961 & 9.991 & 10.001 & 30.001 \\
\hline 8 & 7.566 & 1.894 & 1.933 & 1.992 & 1.261 & 0.499 & 7.881 & 9.801 & 9.971 & 10,000 & 10.001 & 10.001 & 8 & 1.706 & 4.115 & 3.814 & 5.323 & $4.38 \%$ & 2.211 & $5.84 !$ & 9.541 & 9.951 & 9.991 & 10.001 & 10.001 \\
\hline 9 & 4.086 & 1.054 & 9.674 & 1.032 & 1,002 & 2.981 & 0.641 & 9,041 & 9.961 & 0,931 & 10.001 & 10.001 & $y$ & & 2.355 & 1.028 & 2813 & 2.402 & $1.34 !$ & 4371 & $9.23 \mathrm{I}$ & 9.911 & 9.991 & 10,001 & 10.001 \\
\hline 10 & 2076 & 5.605 & 4.589 & 5.033 & 3.382 & $1.73 !$ & 4.971 & 9.3픈 & 9921 & 9.001 & 10.001 & 10.091 & 10 & & 1205 & 9.075 & 1.413 & $1.25 ?$ & 1.422 & 2.881 & 8671 & 9.851 & 9.981 & 10.001 & 10.001 \\
\hline 11 & 1.056 & 2.885 & 2.064 & 2.403 & 1.742 & 9.012 & 3.181 & $87 n 1$ & 9.861 & 9.881 & 10.003 & 10.01 & 11 & & 0.016 & 4.035 & 6.674 & 6223 & 3852 & 1.691 & 7.711 & 9.731 & 9.061 & 9.991 & 10.001 \\
\hline 12 & & 1.435 & 8.735 & 1.093 & 8,203 & 4.392 & 1.761 & 7.681 & 9.721 & 9.961 & 9.991 & 10001 & 12 & & 3.366 & 1.655 & 3.004 & 2963 & $1.91 ?$ & 8962 & 6.2.21 & 4. 481 & 0.031 & 9.991 & 10.001 \\
\hline 13 & & 6.840 & 3.505 & 4734 & 3.753 & 2.0062 & 8.732 & 5.921 & ",d11 & 9.921 & 9.901 & 10.001 & 13 & & 1.006 & 6.0066 & 1.201 & 1.353 & 9.143 & 4.472 & 4.34 & 8.971 & 9.861 & 9.981 & 10.001 \\
\hline 14 & & & 1.325 & $1.96 \mathrm{~d}$ & 1.663 & 9.463 & 4.112 & 3.821 & 8.731 & 4.831 & 9,471 & 14.601 & $1: 9$ & & & & 5.165 & 5.914 & 4233 & 2.152 & 2.591 & 8.001 & 9.721 & 9.961 & 0.901 \\
\hline 15 & & & 4.676 & 7.685 & 7.094 & 4.263 & 1.902 & 2.071 & 7411 & 9.611 & 4.911 & $9.99 \mathrm{I}$ & 15 & & & & 1.965 & $? 464$ & 1.593 & 1.012 & $1.3+1$ & 6.381 & 9.401 & 9.911 & 9.981 \\
\hline 16 & & & 1.586 & 2.855 & 289 & 1.873 & 8.673 & 1.011 & 5.341 & 9.101 & 9811 & 9,481 & 10 & & & & $7.03 \%$ & 9723 & $\$ .11 .4$ & 4583 & 6.712 & 4.311 & 8.721 & 9.801 & 9.971 \\
\hline 17 & & & & 10.006 & 1.124 & 7.924 & 3.003 & 4712 & 3.141 & $7.96 !$ & 9071 & 0,451 & 17 & & & & & 3635 & 3,324 & 2003 & 3.212 & 2.401 & 7.391 & 9.551 & 9.931 \\
\hline 18 & & & & 3.276 & 4.125 & 3.214 & 1.713 & 2.212 & $1.54 !$ & 6021 & 9.191 & $9 \times 1$ & 18 & & & & & 1.295 & 1.204 & 8.584 & 1.522 & 1.261 & 5.371 & 8.971 & 9.831 \\
\hline 19 & & & & & 1.435 & 1.234 & 7.189 & 1.032 & 7.602 & 3.081 & 8.071 & 9051 & 19 & & & & & & 4.765 & 3.474 & 7.033 & 6.192 & 2.251 & 7.751 & 9.581 \\
\hline 20 & & & & & 4676 & 4.485 & 2.884 & 4793 & 3.652 & 1.911 & 0.051 & 9001 & 20 & & & & & & 1.665 & 1.334 & 3.173 & 3.012 & 1721 & 5.761 & 8.981 \\
\hline 21 & & & & & 1.426 & 1.545 & 1.004 & 2.163 & 1.782 & 9332 & $3.67 \mathrm{I}$ & 7711 & 21 & & & & & & 5.426 & 4845 & 1.383 & 1462 & 8672 & 3.551 & 7.701 \\
\hline 22 & & & & & & 4.946 & 3.925 & 9.324 & 8643 & 4.612 & 1.911 & $5,5,1$ & 22 & & & & & & & 1.665 & 5684 & 6923 & 4.352 & 1.911 & 5.621 \\
\hline 23 & & & & & & 1.516 & 1.325 & 3.824 & 4.113 & 2332 & 9572 & 3251 & 23 & & & & & & & 5336 & 2.234 & 3.183 & 2.192 & 9.862 & 3.421 \\
\hline 24 & & & & & & & 4.206 & 1.484 & 1.893 & 1.182 & 4.392 & I foul & 24 & & & & & & & & 8.255 & 1,403 & 1.082 & 5.102 & 1851 \\
\hline 25 & & & & & & & 1.276 & 5.135 & 8244 & 5.883 & 2.562 & 8712 & 25 & & & & & & & & 2845 & 5.854 & 5,233 & 2.652 & 9.752 \\
\hline 26 & & & & & & & & 1.875 & 3.414 & 2.833 & 1.352 & $462 ?$ & 26 & & & & & & & & 9,5006 & 2.314 & 2.423 & 1.372 & 5.202 \\
\hline 27 & & & & & & & & 6000 & 1.339 & 1.303 & 6.903 & 2.512 & 27 & & & & & & & & 2966 & 8.625 & 1.073 & 6.863 & 2.802 \\
\hline 28 & & & & & & & & 1,846 & 4005 & 5.614 & 3.153 & $1366^{\circ}$ & 28 & & & & & & & & & 3035 & 0.444 & 3.313 & 1.492 \\
\hline 29 & & & & & & & & & 1.695 & 2.334 & 1.673 & $7 ? 63$ & 29 & & & & & & & & & & 1.754 & 1.523 & 7.723 \\
\hline 30 & & & & & & & & & 5.980 & 9.025 & 7554 & 37.73 & 30 & & & & & & & & & & 0475 & 6.594 & $3.84 \underline{3}$ \\
\hline 31 & & & & & & & & & 1,696 & 3285 & 3239 & $1, £ 13$ & 31 & & & & & & & & & & 2265 & 2.704 & 1.823 \\
\hline 32 & & & & & & & & & & 1125 & 1304 & 8.691 & 38 & & & & & & & & & & & 1.044 & 8.144 \\
\hline 33 & & & & & & & & & & 3.616 & 4.925 & 3.781 & 33 & & & & & & & & & & & 3.795 & 3.444 \\
\hline 34 & & & & & & & & & & & & 1.571 & 34 & & & & & & & & & & & 1.305 & 1.374 \\
\hline 35 & & & & & & & & & & & & 6195 & 35 & & & & & & & & & & & 4.176 & 5.135 \\
\hline $3 \mathrm{~b}$ & & & & & & & & & & & & 2245 & 36 & & & & & & & & & & & 1.296 & 1815 \\
\hline
\end{tabular}


Table III (Cont'd.)

${ }_{92} \mathrm{U}^{235}$ (TARGET NUCLEUS)

\begin{tabular}{|c|c|c|c|c|c|c|c|c|c|c|c|c|c|}
\hline$\ell$ & 12 & 14 & 16 & 18 & 20 & 22 & 24 & 26 & 30 & 34 & 38 & 42 & 46 \\
\hline 0 & 1.197 & 7.816 & 2.894 & 4.133 & 5.132 & 3.051 & 7.161 & 9.181 & 9.921 & 9.991 & 10.001 & 10.001 & 10.001 \\
\hline 1 & 1.197 & 7.236 & 2.694 & 3.793 & 4.752 & $2.89]$ & 7.011 & 9.121 & 9.921 & 9.991 & 10.001 & 10.001 & 10.001 \\
\hline 2 & 2.988 & $6.27 \underline{6}$ & 2.334 & 3.213 & $4.07 \underline{2}$ & 2.581 & 6.701 & 9.011 & 9.911 & 9.991 & 10.001 & 10.001 & 10.001 \\
\hline 3 & & 5.016 & 1.884 & 2.503 & 3.242 & 2.171 & 6.211 & 8.821 & 9.891 & 9.991 & 10.002 & 10.001 & 10.001 \\
\hline 4 & & 3.766 & 1.424 & 1.803 & 2.382 & 1.701 & 5.521 & 8.521 & 9.861 & 9.981 & 10.001 & 10.001 & 10.001 \\
\hline 5 & & 2.616 & 1.014 & 1.203 & 1.632 & $1.241]$ & 4.641 & $8.04 \underline{1}$ & 9.821 & 9.981 & 10.001 & 10.001 & 10.001 \\
\hline 6 & & 1.696 & 6.765 & 7.394 & 1.042 & 8.342 & 3.611 & 7.331 & 9.741 & 9.971 & 10.001 & 10.001 & 10.001 \\
\hline 7 & & 1.056 & 4.285 & 4.264 & $6.21 \underline{3}$ & 5.222 & $2.58 \mathrm{I}$ & 6.331 & $9.61 \underline{1}$ & 9.951 & 9.991 & 10.002 & 10.001 \\
\hline 8 & & & 2.585 & 2.294 & 3.483 & 3.052 & 1.671 & $5.04]$ & 9.391 & 9.931 & 9.991 & 10.001 & 10.001 \\
\hline 9 & & & 1.485 & 1.154 & $1.84 \underline{3}$ & 1.682 & 9.932 & 3.611 & 9.001 & $9.89 \underline{1}$ & 9.981 & 10.001 & 10.001 \\
\hline 10 & & & 8.116 & 5.445 & 9.224 & 8.763 & 5.472 & 2.291 & 8.311 & 9.811 & 9.971 & 10.001 & 10.001 \\
\hline II & & & 4.246 & 2.415 & 4.374 & $4.37 \underline{3}$ & 2.842 & 1.311 & 7.181 & 9.651 & 9.851 & 9.991 & 10.001 \\
\hline 12 & & & 2.116 & $9.97 \underline{6}$ & 1.964 & $2.08 \underline{3}$ & 1.422 & 6.912 & $5.57 \underline{1}$ & $9.34 \underline{1}$ & 9.911 & 9.991 & 10.001 \\
\hline 13 & & & & 3.906 & 8.335 & 9.504 & 6.783 & 3.452 & 3.731 & 8.731 & 9.831 & 9.971 & 10.001 \\
\hline 14 & & & & & 3.345 & 4.1334 & 3.143 & 1.672 & 2.161 & 7.601 & $9.65 \underline{1}$ & 9.951 & 9.991 \\
\hline 15 & & & & & 1.265 & 1.714 & 1.403 & 7.813 & 1.131 & 5.851 & $9.27 \underline{\underline{I}}$ & 9.891 & 9.981 \\
\hline 16 & & & & & 4.536 & 6.705 & 5.974 & $3.56 \underline{3}$ & $5.54 \underline{\underline{2}}$ & 3.821 & 8.471 & 9.761 & 9.961 \\
\hline 17 & & & & & & 2.485 & 2.424 & 1.563 & 2.662 & 2.141 & 7.001 & 9.461 & 9.911 \\
\hline 18 & & & & & & 8.666 & 9.345 & 6.574 & 1.262 & 1.091 & 4.931 & 8.791 & 9.791 \\
\hline 19 & & & & & & & 3.415 & 2.634 & 5.833 & 5.382 & 2.921 & 7.441 & $9.50 \mathrm{I}$ \\
\hline 20 & & & & & & & $1.17 \underline{\underline{5}}$ & 10.005 & 2.613 & 2.632 & 1.541 & 5.391 & 8.821 \\
\hline 21 & & & & & & & $3.76 \underline{6}$ & 3.595 & 1.123 & $1.27 \underline{2}$ & 7.792 & $3.27 \underline{I}$ & 7.431 \\
\hline 22 & & & & & & & & 1.225 & 4.584 & 5.993 & 3.932 & 1.761 & 5.321 \\
\hline 23 & & & & & & & & 3.886 & 1.784 & 2.733 & 1.972 & 9.102 & 3.201 \\
\hline 24 & & & & & & & & & 6.505 & $1.19 \underline{3}$ & $9.74 \underline{3}$ & 4.732 & 1.731 \\
\hline 25 & & & & & & & & & 2.255 & 4.914 & 4.663 & 2.462 & 9.202 \\
\hline 26 & & & & & & & & & 7.316 & 1.924 & 2.133 & 1.262 & 4.932 \\
\hline 27 & & & & & & & & & & 7.075 & 9.294 & 6.283 & 2.652 \\
\hline 28 & & & & & & & & & & 2.465 & 3.834 & 3.003 & 1.402 \\
\hline 29 & & & & & & & & & & & 1.494 & 1.363 & 7.223 \\
\hline 30 & & & & & & & & & & & 5.455 & 5.844 & 3.563 \\
\hline 31 & & & & & & & & & & & 1.885 & 2.374 & 1.673 \\
\hline 32 & & & & & & & & & & & & 9.045 & 7.384 \\
\hline 33 & & & & & & & & & & & & 3.255 & 3.084 \\
\hline 34 & & & & & & & & & & & & 1.105 & 1.214 \\
\hline 35 & & & & & & & & & & & & 3.526 & 4.505 \\
\hline 36 & & & & & & & & & & & & 1.056 & $1.57 \underline{5}$ \\
\hline
\end{tabular}


Several calculations were made in which all the parameters were fixed, except $\underline{n}$, in the Hill-Ford charge distribution. The results showed that the reaction cross section is rather insensitive to changes in the charge distribution. For a fixed set of Woods-Saxon parameters, the relative cross sections for $22-\mathrm{Mev}$ alpha particles on silver were in the ratio $1.000: 1.000: 1.043$ for $\underline{\mathrm{n}}=20,7.75$ and 3 , respectively.

A. Simplified Calculation of Reaction Cross Sections with a Parabolic Approximation of Optical Model Real Potential

Values of $T_{l}$ and $\sigma_{R}$ have also been calculated with a model $(8)$ which embraces some simplifying assumptions and as a result greatly reduces the computational problem. The potential function given by

$$
V_{\ell}(r)=\frac{2 Z e^{2}}{r}+\frac{\ell(\ell+1)^{2}{ }^{2}}{2 M a r^{2}}-1100 \exp \left[-\left(\frac{r-1.17 A^{1 / 3}}{0.574}\right)\right],
$$

which describes the real part of the barrier [Eq. (5)], is approximated by a parabola. The transmission coefficients $T_{\ell}$ for a parabolic potential $(9)$ are given by

$$
T_{\ell}=\frac{1}{1+\exp \left[\frac{2\left(B_{\ell}-E\right)}{\hbar \omega_{\ell}}\right]}
$$

where $B l$ is the height of the barrier for angular momentum $L, E$ is the center of mass energy, and $\omega_{\ell}$ is the vibrational frequency of the harmonic oscillator having a potential energy function given by the negative of the potential energy function describing the barrier.

The quantity hal in Eq. (6) is fixed by the curvature of the top of the barrier and the reduced mass of the system, and is given by

$$
h \omega_{\ell}=\left|\frac{\hbar^{2}}{M_{\alpha}} \frac{d^{2} V_{\ell}}{d r^{2}}\right|^{1 / 2}
$$

where $\mathrm{d}^{2} V_{l} / \mathrm{dr} \mathrm{r}^{2}$ is the second derivative of the real part of the barrier (Eq.5) evaluated for the value of $\underline{r}$ for which $V l(r)$ is a maximum.

B. Comparison of Reaction Cross Sections from Various Models

The alpha-particle barriers of $\mathrm{U}^{235}$ for the square-well potential, optical-model potential, and the parabolic approximation to the real part of 
the optical-model potential are plotted in Fig. 1 for $l=0$ and in Fig. 2 for $\ell=25$. The square-well potential (curve a) includes the Coulomb and centrifugal barriers and a constant nuclear potential of $-50 \mathrm{Mev}$ for $r<\left(1.50 \mathrm{~A}^{1 / 3}+1.21\right)$ fermi and 0 for $r>\left(1.50 \mathrm{~A}^{1 / 3}+1.21\right)$ fermi.

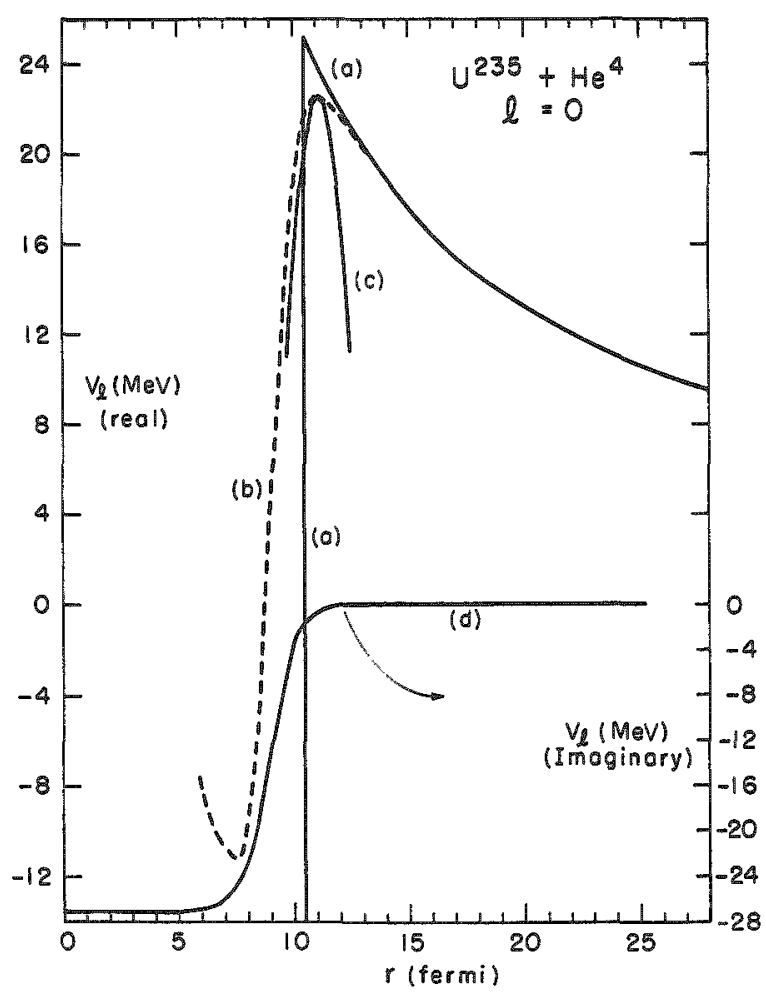

Fig. 1

Potential barriers for the reaction ${ }_{92} \mathrm{U}^{235}+{ }_{2} \mathrm{He}^{4}$ for angular momentum $\ell=0$. (a) square-well potential for radius equal to $\left(1.50 \mathrm{~A}^{1 / 3}+1.21\right)$ fermi and a constant nuclear potential of $V=-50 \mathrm{Mev}$ for $r<\left(1.50 \mathrm{~A}^{1 / 3}+1.21\right)$ fermi and $V=0$ for $r>\left(1.50 A^{1 / 3}+1.21\right)$ fermi. (b) Opticalmodel potential (real part) given by Eq. (2) with a nuclear potential equal to $-50 /\left[1+\exp \left(\frac{r-1.17 A^{1 / 3}-1.77}{0.576}\right)\right]$

(c) Parabolic approximation of the real part of the optical-model potential [see Eqs. (5), (6), and (7) in text]. (d) Imaginary part of the optical-model potential which is given by $-27 i /\left[1+\exp \left(\frac{x-1.17 A^{1 / 3}-1.77}{0.576}\right)\right]$. 


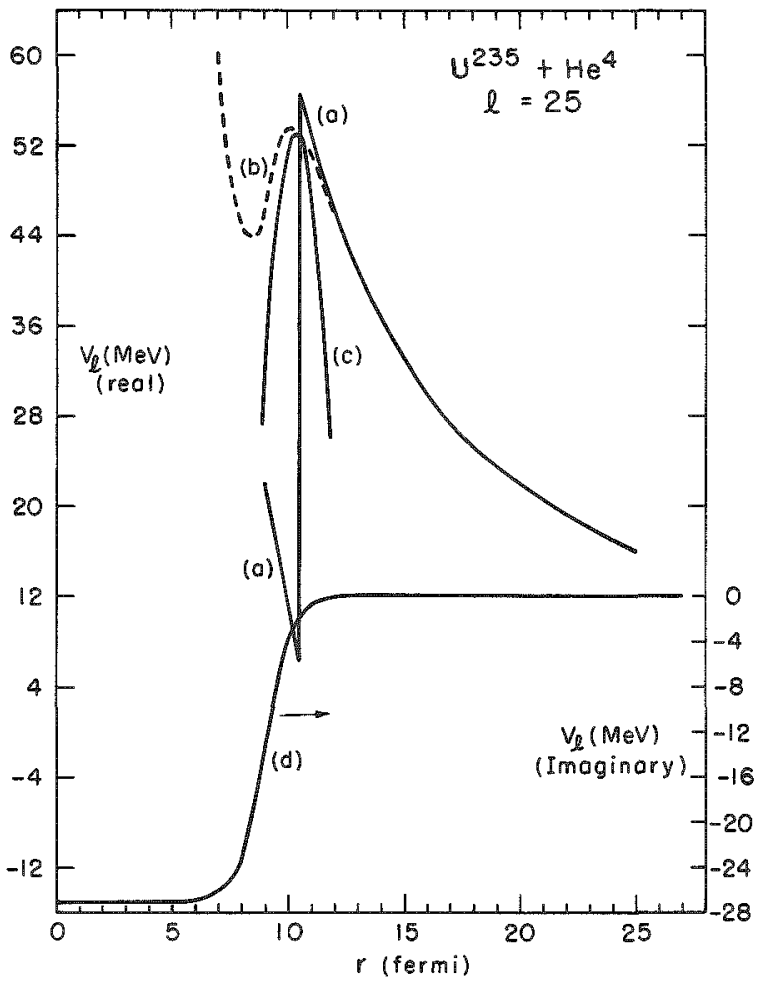

Fig. 2

Potential barriers for the reaction ${ }_{92} \mathrm{U}^{235}+{ }_{2} \mathrm{He}^{4}$ for angular momentum $\ell=25$. See caption of Fig. 1 for description.

The real part of the optical-model potential for uranium (curve b) is calculated from Eq. (2) where the nuclear potential is given by

$$
-50 /\left[1+\exp \left(\frac{r-1.17 \mathrm{~A}^{1 / 3}-1.77}{0.576}\right)\right] \text {. }
$$

The imaginary part of the optical model potential for uranium (curve d) is given by

$$
-27 i /\left[1+\exp \left(\frac{r-1.17 A^{1 / 3}-1.77}{0.576}\right)\right]
$$

and these calculated values are also plotted in the above figures. The parabolic approximation to the real part of the optical-model potential (curve c) is calculated from Eqs. (5) and (7).

The maximum in the real part of the optical-model potential moves to smaller radii for increasing values of the angular momentum, whereas the imaginary part of the optical-model potential is independent of angular momentum. Due to this effect and the strong alpha-particle absorption, the waves with larger $\&$ values experience a smaller fraction of the width of the barrier than do the waves with small $\ell$ values. This means that the alpha particle is absorbed in some cases before it traverses the entire real part of the optical-model potential, i.e., absorption occurs in the 
barrier and the alpha particle does not reach the radius corresponding to the inner turning point of the barrier. Such a model leads to $T$ l values which may be larger than those calculated from models in which it is as sumed that the alpha particle is not absorbed in the barrier region.

The reaction cross sections obtained with $E q .(1)$ for the squarewell potential, $(1,2)$ the optical-model potential, and the parabolic approximation to the real part of the optical model potential are plotted for comparison in Fig. 3 for a ${ }_{23} \mathrm{~V}^{51}$ target and in Fig. 4 for a ${ }_{92} \mathrm{U}^{235}$ target. The square-well potential with $r=\left(1.5 \mathrm{~A}^{1 / 3}+1.21\right)$ fermi gives reaction cross sections which are smaller by a factor of two than the opticalmodel values. Approximate agreement between the square-well and optical-model cross sections can be obtained for larger values of the square-well radius. If the value of $\triangleq$ in the square-well radius, $r=r_{0} A^{1 / 3}+\Delta$, is 1.2 fermi, then $r_{0}$ must be equal to 1.63 fermi for uranium and 1.9 fermi for vanadium in order to give agreement. Stated in another way, if $r_{0}$ in the square-well radius is 1.5 fermi, then $\Delta$ must be equal to 2.0 fermi for uranium and 2.7 fermi for vanadium in order that the square-well model gives cross sections as large as the optical model.

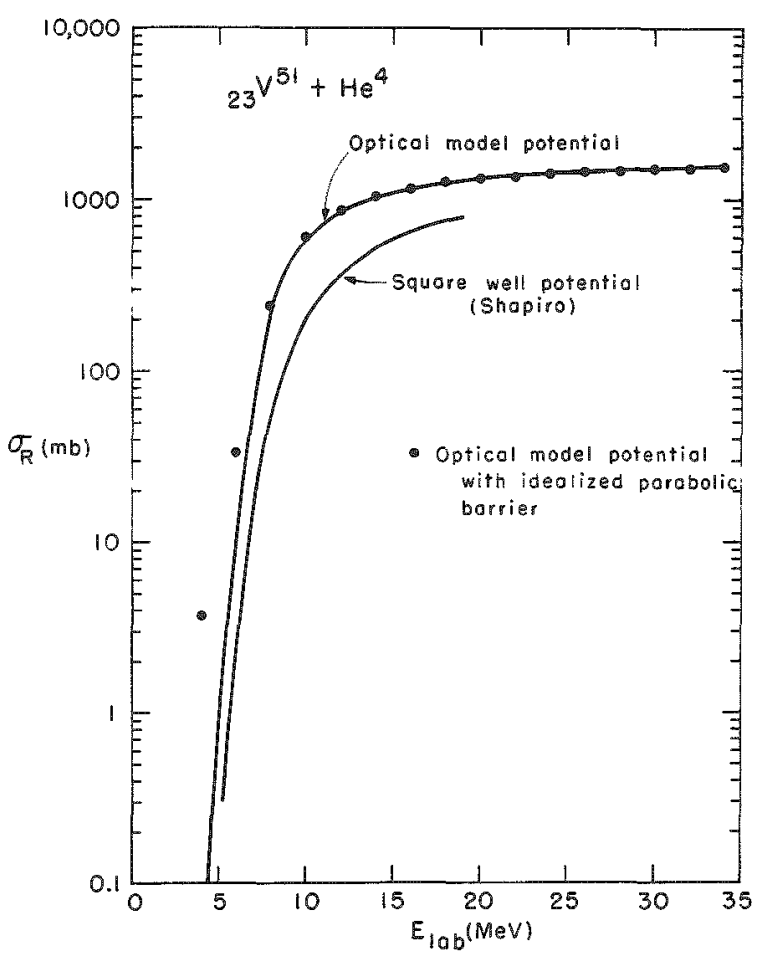

Fig. 3

Reaction cross sections for ${ }_{23} \mathrm{~V}^{51}+{ }_{2} \mathrm{He}^{4}$ are plotted versus the alpha-particle energy in the laboratory system.

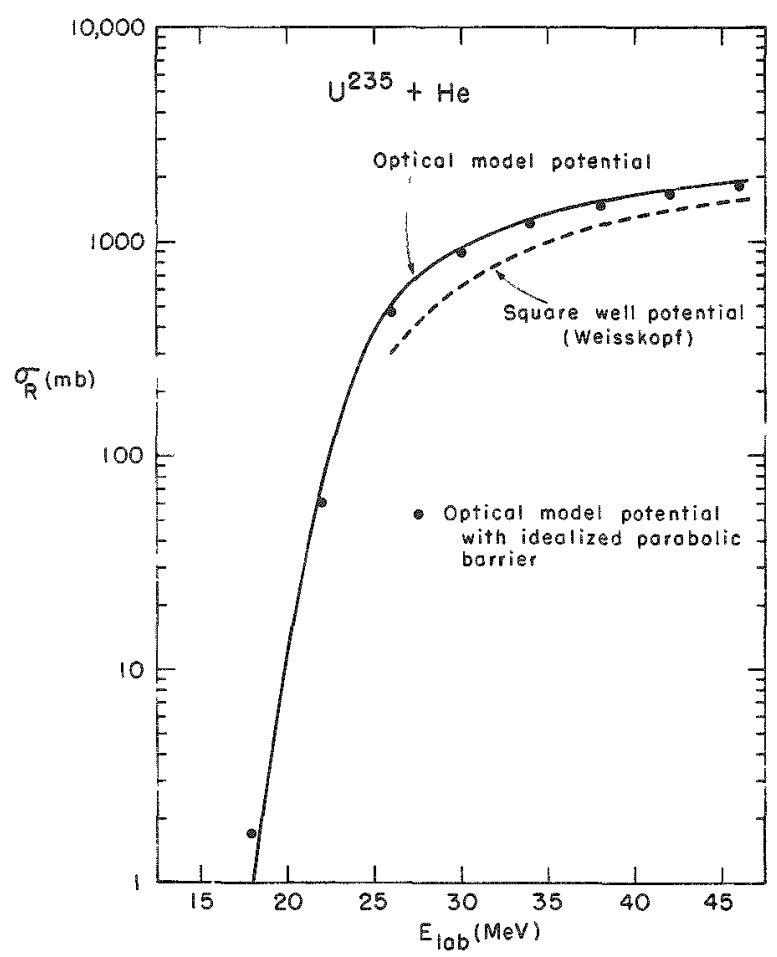

Eig. 4

Reaction cross sections for ${ }_{92} \mathrm{U}^{235}+{ }_{2} \mathrm{He}^{4}$ are plotted versus the alpha-particle energy in the laboratory system. 
A square-well radius of $\left(1.5 \mathrm{~A}^{1 / 3}+2.2\right)$ fermi will give reaction cross sections which agree reasonably well with optical-model cross sections for a number of elements.

The agreement between the reaction cross sections derived from the optical model and from a model which approximates the real part of the optical-model potential by a parabola and utilizes the transmission coefficients for a parabolic potential is very good for energies exceeding the classical barriex height $(\hat{h}=0)$. The degree of agreement in cross sections for vanadium with alpha-particle bombarding energies greater than $8 \mathrm{Mev}$ and for uranium with energies greater than $20 \mathrm{Mev}$ can be seen in Figs. 3 and 4, respectively. At energies below the classical barrier, the agreement between the transmission coefficients and reaction cross sections derived from the two models is unsatisfactory. The merit of the model which utilizes the parabolic approximation of the real part of the optical-model potential lies, of course, in the relatively simple mathematical computation which is necessary for calculating rather accurate reaction cross sections.

The ratios of the reaction cross section for angular momentum $\frac{\ell}{5}$ to the total reaction cross section, $\sigma_{\mathrm{R}} / \sigma_{\mathrm{R}}$, are plotted against $\hat{z}$ in $\mathrm{Fig} . \overline{5}$ for 12 - Mer (Lab) alpha particles on ${ }_{23} \mathrm{~V}^{51}$ for the three models discussed above. Reasonably good agreement is obtained between the optical- and parabolic-potential models. This results from the fact that the cross sections and the individual transmission coefficients are approximately equal for these two models at all energies greater than the classical barrier.

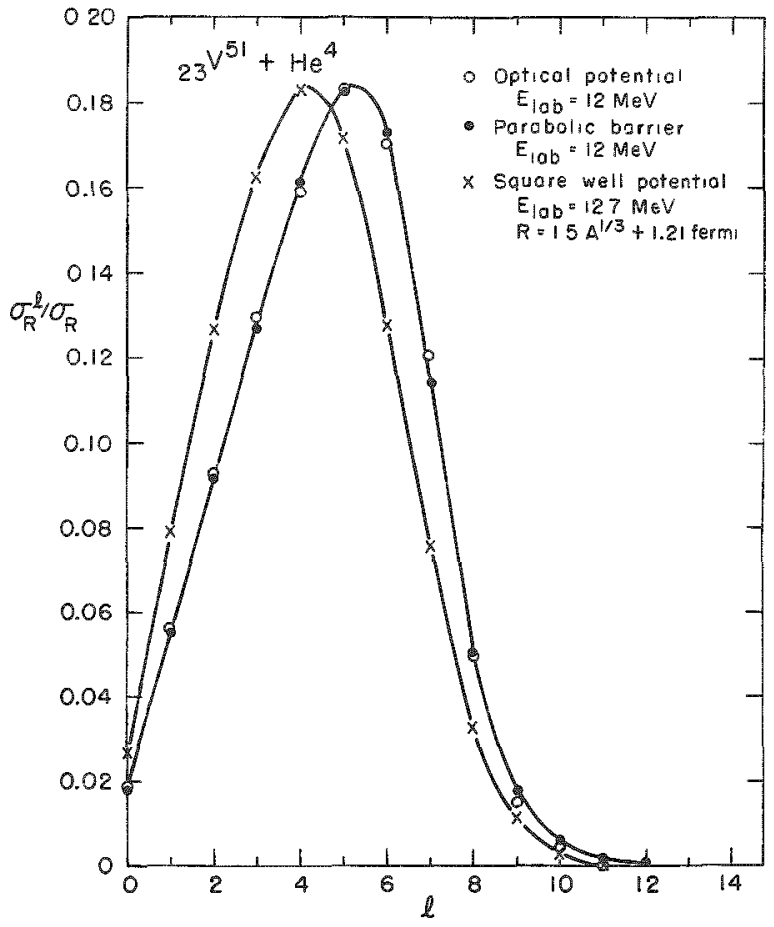

Fig. 5

The ratios of the reaction cross section for angular momentum $\ell$ to the total reaction cross section, $\sigma_{\mathrm{R}} / \sigma_{\mathrm{R}}$. are plotted versus $\&$ for $12-\mathrm{MeV}$ (Lab) alpha particles on ${ }_{23} \mathrm{~V}^{51}$ for the three models discussed in the text. 
The square-well model $\left(r=1.5 \mathrm{~A}^{1 / 3}+1.2\right)$ gives considerably smaller average angular momentum deposited in that the reaction cross sections are less by a factor of two. If the square-well radius is increased to give agreement between the square-well and optical-model cross sections, then the average angular momentum deposited in the square-well model is greater than in the optical model. The square-well model leads to considerable reflection from the spike in the potential barrier and, in order to reproduce the magnitude of the optical-model cross sections, more? waves are required in the square-well model.

C. Optical-model Cross-section Dependence on Nuclear Potential

This section is concerned with an investigation of the dependence of the reaction cross section on the nuclear potential in the optical-model calculation.

The first comparison of reaction cross sections in this section are for different sets of Woods-Saxon parameters which give equally good fits to the complex nuclear potential of $\mathrm{Eq}$. (2) for values of $\underline{x}$ where the real part of the nuclear potential is greater than $-10 \mathrm{Mev}$. The relevant data are summarized in Table IV. The two sets of parameters for uranium (1) $V=-50 \mathrm{Mev}, W=-27 \mathrm{Mev}$, and $r=8.99$ fermi, and (2) $\mathrm{V}=-25 \mathrm{Mev}$, $W=-13.9 \mathrm{Mev}$ and $r^{\circ}=9.39$ fermi, give approximately the same complex nuclear potential for values of the radius greater than 10 fermi. The reaction cross sections for these two different sets of Woods-Saxon parameters agree very well at both 22 and $38 \mathrm{Mev}$. Similar cross-section comparisons are made for vanadium. The two sets of Woods-Saxon parameters in Table IV do not give quite as good agreement in the reaction cross-section values for the lighter nucleus, ranadium.

\section{Table IV}

REACTION CROSS-SECTION DEPENDENCE ON VARIOUS SETS OF WOODS-SAXON PARAMETERS WHICH GIVE APPROXIMATELY THE SAME COMPLEX NUCLEAR POTENTIALS FOR $\mathrm{r}$ VALUES WHERE THE REAL PART OF THE NUCLEAR POTENTIAL IS GREATER THAN - $10 \mathrm{MEV}$.*

\begin{tabular}{|c|c|c|c|c|c|c|}
\hline Nuclide & $\begin{array}{c}\mathrm{E}_{\text {Lab }} \\
(\text { Mev })\end{array}$ & $V($ Mev $)$ & $W($ Mev $)$ & $\mathrm{r}_{0}$ (fermi) & $\mathrm{n}$ & $\sigma_{\mathrm{R}}(\mathrm{mb})$ \\
\hline${ }_{23} \mathrm{~V}^{51}$ & 6 & -50 & -10 & $1.17 \mathrm{~A}^{1 / 3}+1.77$ & 0.04 & 10.5 \\
& 6 & -25 & -5.2 & $1.17 \mathrm{~A}^{1 / 3}+2.17$ & 6.04 & 10.6 \\
& 34 & -50 & -10 & $1.17 \mathrm{~A}^{1 / 3}+1.77$ & 0.04 & 1559 \\
& 34 & -25 & -5.2 & $1.17 \mathrm{~A}^{1 / 3}+2.17$ & 6.04 & 1492 \\
& 22 & -50 & -27 & $1.17 \mathrm{~A}^{1 / 3}+1.77$ & 10.04 & 76.8 \\
& 22 & -25 & -13.9 & $1.17 \mathrm{~A}^{1 / 3}+2.17$ & 10.04 & 77.2 \\
& 38 & -50 & -27 & $1.17 \mathrm{~A}^{1 / 3}+1.77$ & 10.04 & 1550 \\
& 38 & -25 & -13.9 & $1.17 \mathrm{~A}^{1 / 3}+2.17$ & 10.04 & 1552 \\
\hline
\end{tabular}

* In all cases

and

$$
d(\operatorname{fermi})=0.576
$$

$r_{c}(\operatorname{fermi})=1.17 \mathrm{~A}^{1 / 3}$ 
The result of comparisons in Table IV confirms earlier suggestions that the depth of the potential for small $r$ values is unimportant in analyzing alpha-particle elastic scattering and total reaction cross-section data as long as the set of parameters in the Woods-Saxon potential accurately reproduces the complex potential near the nuclear surface.

Whereas the cross-section comparisons in Table IV are for sets of optical-model parameters which give approximately the same complex potential at $x$ values larger than the nuclear radius (although considerably different complex potentials at small values of $r$ ), the comparisons in Table $\mathrm{V}$ are for sets of optical-model parameters which give different complex nuclear potentials for large values of $\underline{x}$.

Table $V^{7}$

REACTION CROSS-SEC IION DEPENDENCE OA THE COMPLEX NUCLEAR POTENTIAL*

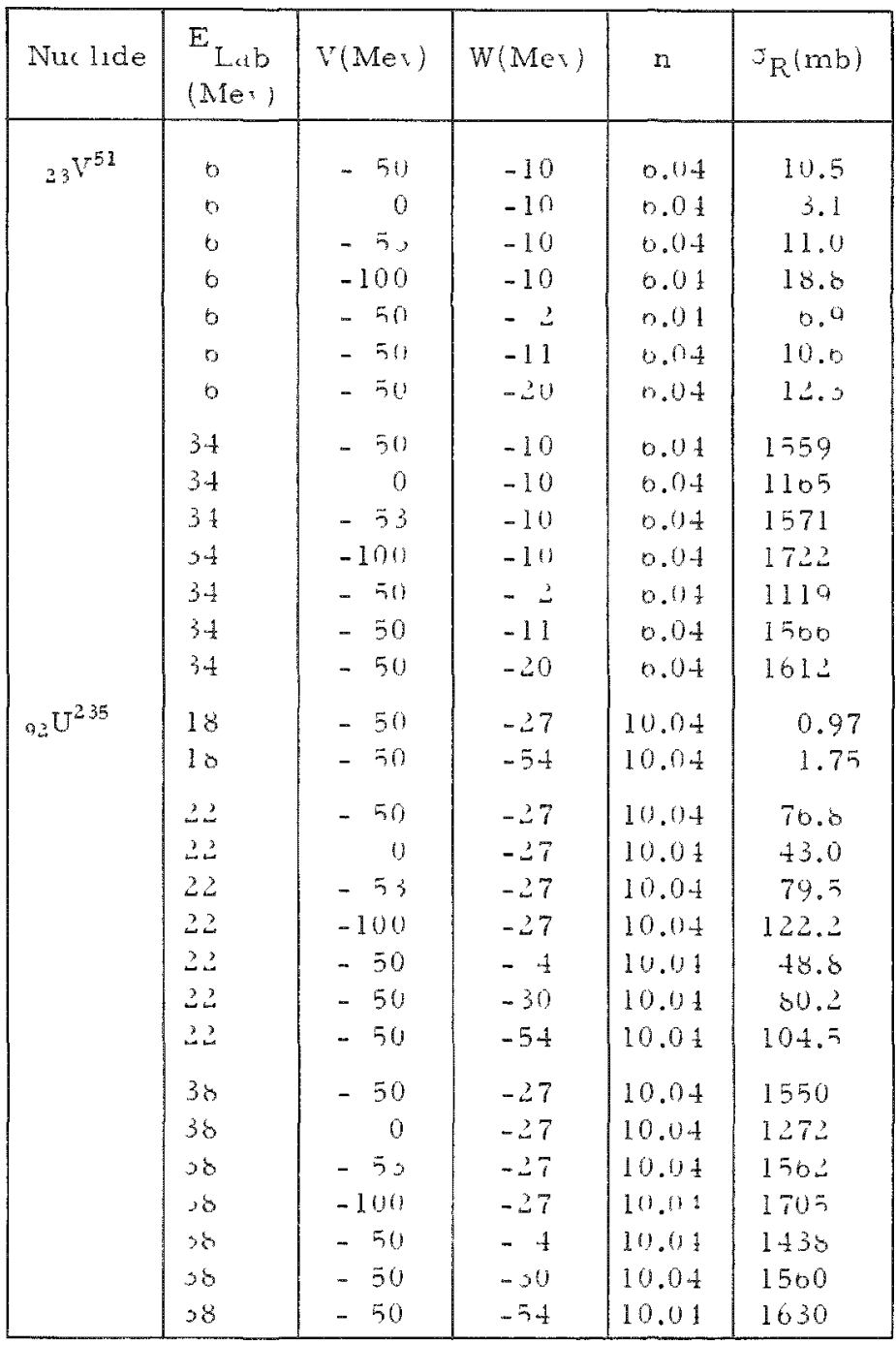

* In all cases

$$
\begin{aligned}
& \text { reftermi }=1.17 \mathrm{~A}^{2}+1.77 \\
& \text { d(terms) }=0.570 \\
& r_{c}(\operatorname{term} 1)=1.17 \mathrm{~A}^{13}
\end{aligned}
$$


The first entry for each energy in Table $V$ gives the appropriate cross section for the Woods-Saxon parameters of Table I, which are based on the experimental nuclear potential (4)

$$
-1100 \exp \left[-\left(\frac{r-1.17 \mathrm{~A}^{1 / 3}}{0.574}\right)\right]-45.7 \mathrm{i} \exp \left[-\left(\frac{x-1.40 \mathrm{~A}^{1 / 3}}{0.578}\right)\right]
$$

for large $\underline{\underline{r}}$ values (i.e., values of $\underline{\underline{r}}$ where the real part of the potential is greater than $-10 \mathrm{Mev}$ ). The remainder of the entries for each energy are for various values of the complex nuclear potential. The parameters $V$ and $W$ are varied separately with the other Woods-Saxon parameters fixed such that the change in the complex nuclear potential at large values of $\underline{r}$ is proportional to the change in $\underline{V}$ or $\underline{W}$. For example, the set of Woods-Saxon parameters for $U^{235}(\mathrm{~V}=-100 \mathrm{Mev}, \mathrm{W}=-27 \mathrm{Mev}$, $r_{0}-1.17 A^{1 / 3}+1.77$, etc.) correspond to a complex nuclear potential equal to

$$
-2200 \exp \left[-\left(\frac{r-1.17 A^{1 / 3}}{0.574}\right)\right]-45.7 i \exp \left[-\left(\frac{r-1.40 A^{1 / 3}}{0.578}\right)\right]
$$

for large values of the radius, or a change of a factor of two in the real part of the nuclear potential. Since the uncertainty in the real part of the nuclear potential as determined by analyses (4) of the elastic-scattering data is thought to be approximately $50 \%$, the above value of the real potential is outside the limit of this error.

The uncertainty in the imaginary part of the nuclear potential as determined by alpha-particle elastic scattering is, however, considerably larger than the uncertainty in the real part of the nuclear potential, and may be as much as $100 \%$ or even larger. The set of parameters for $\mathrm{U}^{235}$ $\left(\mathrm{V}=-50 \mathrm{Mev}, \mathrm{W}=-54 \mathrm{Mev}, \mathrm{r}_{0}=1.17 \mathrm{~A}^{1 / 3}+1.77\right.$, etc.) corresponds to a complex nuclear potential equal to

$$
-1100 \exp \left[-\left(\frac{r-1.17 A^{1 / 3}}{0.574}\right)\right]-91.4 \mathrm{i} \exp \left[-\left(\frac{r-1.40 \mathrm{~A}^{1 / 3}}{0.578}\right)\right]
$$

at the nuclear surface, which represents a change of a factor of 2 in the imaginary part of the nuclear potential from the average value(4) deduced from elastic scattering.

At energies considerably larger than the classical barrier, the cross sections vary only slightly with large variations in either the real or imaginary part of the nuclear potential. Variation of either the real or imaginary potential by a factor of 2 at large values of $\underline{r}$ changes the reaction cross section by only $10 \%$ or less. In the region of the Coulomb barrier, however, the reaction cross sections vary considerably with large changes in the complex nuclear potential. The strong dependence of the reaction cross section on the real and imaginary potentials at large values of $r$ for energies near the Coulomb barrier can be seen from the 
data of Table V. For the target nucleus $U^{235}$ a change in $W$ from -27 to $-5+\mathrm{Mer}$ increases the reaction cross section by $36 \%$ for $\overline{22}-\mathrm{Mev}$ alpha particles and by $80 \%$ for $18-\mathrm{Mev}$ alpha particles. Since reaction crosssection values are very sensitive to the complex nuclear potential at energies below the classical barrier, accurate experimental measurements of the reaction cross sections for alpha particles at these energies in conjunction with the alpha-particle elastic-scattering data would be useful in the determination of the complex nuclear potential.

D. Program Checks

The results of our program were checked with data published by Glassgold for 10-Mev protons (10) on argon and copper and for $22-\mathrm{Mer}$ helium ions on silver.(11) Some of the other quantities recorded in our program are the Coulomb functions and the real and imaginary parts of the nuclear phase shifts for each $\underline{\ell}$. The authors will supply any of this information to those interested on request.

\section{ACKNOWLEDGMENTS}

One of the authors (JRH) wishes to thank Professors I. Perlman and $\mathrm{J}$. O. Rasmussen for their hospitality during his summer visit at the Lawrence Radiation Laboratory in Berkeley. We also wish to thank J. O. Rasmussen, R. Griffioen, and R. Vandenbosch for several helpful discussions. 
1. M. M. Shapiro, Phys.Rev.90, 171 (1953).

2. J.M. Blatt and V.F. Weisskopf, Theoretical Nuclear Physics (John Wiley and Sons, Inc., New York, 1952), p. 352.

3. H. Feshbach, M. M. Shapiro, and V.F. Weisskopf, NYO-3077, unpublished.

4. G. Igo, Phys. Rev. 115, 1665 (1959).

5. K. W. Ford and D. L. Hill, Phys。Rev. 94, 1617 (1954).

o. R. Hofstadter, Annual Review of Nuclear Science (Annual Reviews, Inc., Palo Alto, California, 1957), Vol. 7, p. 231.

7. R. D.Woods and D.S. Saxon, Phys.Rev. 95, 577 (1954).

8. Suggested by J. O. Rasmussen and programmed by T. D. Thomas, Phys. Rev。116, 703 (1959).

9. D. L. Hill and J. A. Wheeler, Phys.Rev. 89, 1102 (1953), in particular, p. 1140 .

10. A. E. Glassgold, W. B. Cheston, M. L. Stein, S. B. Schuldt, and G.W.Erickson, Phys。Rev。106, 1207 (1957).

11. W. B. Cheston and A. E. Glassgold, Phys.Rev. 106, 1215 (1957). 